\title{
Wedge-local observables for factorizing S-matrix with gap in the coupling constant
}

\author{
Daniela Cadamuro \\ e-mail: daniela.cadamuro@mathematik. uni-goettingen.de \\ Mathematisches Institut, Universität Göttingen \\ Bunsenstrasse 3-5, D-37073 Göttingen, Germany. \\ Yoh Tanimoto \\ e-mail: hoyt@mat.uniroma2.it \\ Dipartimento di Matematica, Università di Roma "Tor Vergata" \\ Via della Ricerca Scientifica 1, 00133 Roma, Italy
}

\begin{abstract}
In the bootstrap approach to integrable quantum field theories in the $(1+1)$ dimensional Minkowski space, one conjectures the two-particle S-matrix and tries to study local observables. We find a family of two-particle S-matrices parametrized by two positive numbers, which are separated from the free field or any other known Smatrix. We propose candidates for observables in wedge-shaped regions and prove their commutativity in the weak sense.

The sine-Gordon model is conjectured to be equivalent to the Thirring model, and its breather-breather S-matrix components (where the first breather corresponds to the scalar field of the sine-Gordon model) are closed under fusion. Yet, the residues of the poles in this breather-breather S-matrix have wrong signs and cannot be considered as a separate model. Our S-matrices differ from the breather-breather S-matrix in the sine-Gordon model by CDD factors which adjust the signs, so that this sector alone satisfies reasonable assumptions.
\end{abstract}

Dedicated to Karl-Henning Rehren on the occasion of his 60th birthday

\section{Introduction}

Recently there have been progresses in the construction of $(1+1)$-dimensional quantum field theories with factorizing S-matrices in the operator algebraic approach [Lec03, Lec08, DT11, Tan12, BT13, LST13, LS14, Tan14, Ala14, BT15, AL16]. The basic idea is the following [Sch97]: while pointlike local observables are hard to construct, observables localized in an infinitely extended wedge-shaped region might be tractable and have simple expressions. It has been first implemented for a scalar analytic factorizing S-matrix [Lec08, Ala14, AL16] and strictly local observables have been shown to exist using a quite indirect proof that 
relies on properties of the underlying modular operators (for double cones above the minimal size). In this construction, the input is the particle spectrum of the theory, together with the S-matrix with certain properties. Construction of observables in wedges has been extended to theories with several particle species by Lechner and Schützenhofer [LS14], including the $O(N)$-invariant nonlinear $\sigma$-models.

Recently, in [CT15, CT16, Tan16], we further generalized this construction to scalar models with S-matrices which have poles in the physical strip. The poles in the S-matrix are believed to correspond to the presence of bound states (e.g. the Bullough-Dodd model). We also extended this construction to models with several particle species, where the S-matrix is "diagonal" in a certain sense. They include, e.g. the $Z(N)$-Ising model and the $A_{N}$-affine Toda field theories.

In this work, we extend this last mentioned class of S-matrices to a continuous family parametrized by two positive numbers. They differ from the S-matrix of the sine-Gordon model by a CDD factor. This has the same fusing table as that of two breathers in the sine-Gordon model.

The sine-Gordon model ${ }^{1}$ has been constructed by Park [Par77] and conjectured to be equivalent to the Thirring model in a certain sense (Coleman's equivalence). In [BFM09] Benfatto, Falco and Mastropietro proved the equivalence between the sine-Gordon model with finite volume interaction and the Thirring model with a finite volume mass term. The Thirring model has been also constructed by the functional integral methods [BFM07]. On the other hand, the sine-Gordon model has been expected to be integrable and its S-matrix has been conjectured [ZZ79]. Yet, in the rigorous constructions, the factorization of the Smatrix has not been proved (c.f. [BR16], where the perturbative S-matrix with IR cutoff is shown to converge, yet its factorization has not been proved).

The conjectured S-matrix of the sine-Gordon model has been studied in the form factor programme [BFKZ99, BK02]. Certain matrix components of the pointlike local fields ("form factors") have been computed, yet the existence of the Wightman field is currently out of reach, because the expansion of the $n$-point functions in terms of form factors is not under control. Here, we are not dealing with the sine-Gordon model itself, but with a new model with the same fusion structure, that we have not considered before. It arises as a modification of the "breather-breather" S-matrix of the sine-Gordon model by the multiplication of a CDD factor. The coupling constant is restricted here to a certain range of values, where there are only two species of particles involved (two breathers). The breather sector for the S-matrix of the sine-Gordon model has been extensively studied in the form factor programme, see e.g. [BK02] and references therein. Yet, the residues of the breather-breather S-matrix have the wrong sign, and they must be contained in the larger model with solitons (the Thirring model). Instead of it, we modify the breather-breather S-matrix by a CDD factor which corrects the sign. In this way, there is no obvious obstruction to relate directly (without

\footnotetext{
${ }^{1}$ In some papers where the model was rigorously constructed, this model is called the "massless" sineGordon model. This refers to the fact that the Lagrangian $\mathcal{L}=\frac{1}{2}\left(\partial_{\mu} \phi\right)^{2}+\frac{m_{0}^{2}}{\beta^{2}} \cos (\beta \phi)$ of the model does not contain the mass term in the standard form $m^{2} \phi^{2}$ in the kinetic part (even if such a term arise from the expansion of the interaction term). The resulting theory, however, is believed to obtain an isolated mass shell. The model corresponding to the Lagrangian with non-zero mass term has been first constructed and the existence of a bound state has been proved [FS76].
} 
adding solitons) these S-matrices with a local quantum field theory ${ }^{2}$.

Indeed, we aim at a realization of this model associated with this new S-matrix in the operator-algebraic framework, i.e. the Haag-Kastler axioms. In this framework, we construct candidates for wedge-local observables by extending the construction carried out in [CT16], see also [Lec15, Section 3] for a general overview. As mentioned before, this is a first step in the construction of strictly local observables, which would be recovered subsequently, following this program, by taking intersection of the algebras generated by observables in right and left wedges, and by using an abstract argument based on a certain phase space property called modular nuclearity. The question of strong commutativity of these wedgelocal observables remains open also in this model.

With the presence of poles in the S-matrix, the construction of wedge-local observables must be studied in a case-by-case approach, in contrast to the homogeneous construction for the analytic S-matrices [LS14]. This is due to the idea that simple poles in the S-matrix correspond to the bound states in the model, therefore, the wedge-local observables must reflect such fusing processes. We do this by introducing the operators which we call the bound state operators. Furthermore, higher order poles bring further complications and we need the existence of what we call elementary particles. Our proof of wedge-locality is based on a number of properties of the two-particle scattering function, and there is actually a infinite family of examples satisfying them, therefore, we have correspondingly an infinite family of candidates for quantum field theories.

As our work proceeds case-by-case, we warn the reader that the properties of the Smatrix we assume are not generic. For example, any model whose S-matrix components of the elementary particle (the particle which generates the whole particle spectrum by fusing process) contain double or higher poles are beyond the reach of our present methods.

The paper is organized as follows. In Sec. 2, we will introduce the model and fix the input scattering data, including the properties of the S-matrix. In Sec. 3, we exhibit our general notation for multi-particle Fock space, partially following Lechner-Schützenhofer [LS14]. In Sec. 4 we introduce the bound state operators $\chi(f), \chi^{\prime}(g)$, we analyse their domains and symmetry properties as quadratic forms. In Sec. 5 we construct the fields $\widetilde{\phi}(f)$ and $\widetilde{\phi^{\prime}}(g)$ and show the weak wedge-commutativity between the components for "elementary particles". In Sec. 6 we conclude our paper with some remarks.

\section{The S-matrix with gap in the coupling constant}

\subsection{The factorizing S-matrix}

The S-matrix describes the result of scattering in the asymptotic time. From physical requirements on quantum field theory, it follows that S-matrix must satisfy several properties, but the most general ones are not enough to specify the S-matrix. In several models, the $\mathrm{S}$-matrix is conjectured to be factorizing and additional properties are conjectured for specific models.

\footnotetext{
${ }^{2}$ Let us note that this does not mean that there is a classical Lagrangian corresponding to these S-matrices. Actually, the family cannot be connected to the free field in a natural way, therefore, it is difficult to relate our models to the free field by perturbation.
} 
On the other hand, our goal is to construct Haag-Kastler nets having a given function as the two-particle scattering function. For this purpose, we also need that the given function satisfies certain properties. Yet, as we are not aiming at constructing models with Lagrangian, these properties might differ from those known in the literature. They are rather justified by the fact that we can construct wedge-observables satisfying the weak commutativity, hence opening the possibility for the full Haag-Kastler net.

Our S-matrix is inspired by the sine-Gordon S-matrix and they differ only by an analytic factor, hence let us give a brief overview of the latter. In the conjectured integrable sineGordon model, the particle spectrum consists of a family of finitely many particles called breathers $\left\{b_{\ell}\right\}$ [BK02]. It is also conjectured that, the sine-Gordon model is equivalent to the Thirring model, where the breathers are the bound states of soliton and the anti-soliton (the anti-particle of the soliton).

In the sine-Gordon model, the number of breathers depends on the coupling constant $0<\nu<1$ in the expression of the Lagrangian [BK02]. We will consider the coupling constant in the interval $\frac{2}{3}<\nu<\frac{4}{5}$, and differently from the sine-Gordon model, we do not consider solitons and interpret that there are only two breathers $b_{1}, b_{2}$, by taking the $\max$ imal analyticity (see below) in a strict sense. ${ }^{3}$ The masses of the breathers are given by $m_{b_{\ell}}=2 m \sin \frac{\ell \nu \pi}{2}$, where $m>0$ and $\ell=1,2$. These particles are neutral and hence the charge conjugate of $b_{\ell}$ (denoted with $\bar{b}_{\ell}$ in literature) is $b_{\ell}$ itself. The elastic two-particle scattering processes are characterized by a matrix-valued function with only non-zero components $S_{b_{1} b_{1}}^{\mathrm{SG}}(\theta), S_{b_{2} b_{1}}^{\mathrm{SG}}(\theta), S_{b_{1} b_{2}}^{\mathrm{SG}}(\theta)$ and $S_{b_{2} b_{2}}^{\mathrm{SG}}(\theta)$, where $\theta$ is the difference of the rapidities of the incoming particles.

Our new S-matrix has the same particle spectrum as the breather-breather sector of the sine-Gordon model, and admit components, $S_{b_{1} b_{1}}(\theta), S_{b_{2} b_{1}}(\theta), S_{b_{1} b_{2}}(\theta)$ and $S_{b_{2} b_{2}}(\theta)$, similarly to those of the sine-Gordon model. We will give explicit expressions for them in Section 2.2. The difference between $S_{b_{k} b_{\ell}}(\theta)$ and $S_{b_{k} b_{\ell}}^{\mathrm{SG}}(\theta)$ is another analytic function called CDD factors.

The particles $b_{1}, b_{2}$ may form a bound state in a scattering process. We declare that the possible fusing processes are only of three types, $\left(b_{1} b_{1}\right) \rightarrow b_{2},\left(b_{1} b_{2}\right) \rightarrow b_{1}$ and $\left(b_{2} b_{1}\right) \rightarrow b_{1}$. On the other hand, $\left(b_{2} b_{2}\right)$ is not a fusion. The corresponding imaginary rapidities of the fusing particles are denoted by $\theta_{\left(b_{1} b_{1}\right)}^{b_{2}}$ for the first fusion, and $\theta_{\left(b_{1} b_{2}\right)}^{b_{1}}, \theta_{\left(b_{2} b_{1}\right)}^{b_{1}}$ for the second two types of fusion. Correspondingly, we do not specify the rapidity $\theta_{\left(b_{2} b_{2}\right)}$, since there is no fusion $\left(b_{2} b_{2}\right)$. The actual values will be given in Section 2.2.

In the same way as in [CT16], to these fusing processes there correspond the so-called fusion angles, which determine the position of the simple poles in the components $S_{b_{1} b_{1}}(\zeta)$, $S_{b_{1} b_{2}}(\zeta)$ and $S_{b_{2} b_{1}}(\zeta)$ in the physical strip $0<\operatorname{Im} \zeta<\pi$. Specifically, for the fusion $\left(b_{1} b_{1}\right) \rightarrow b_{2}$, $S_{b_{1} b_{1}}(\zeta)$ has a simple pole at $\zeta=i \theta_{b_{1} b_{1}}^{b_{2}}$, where

$$
\theta_{b_{1} b_{1}}^{b_{2}}:=\theta_{\left(b_{1} b_{1}\right)}^{b_{2}}+\theta_{\left(b_{1} b_{1}\right)}^{b_{2}}\left(=2 \theta_{\left(b_{1} b_{1}\right)}^{b_{2}}\right) .
$$

Similarly, $S_{b_{1} b_{2}}(\zeta)$, corresponding to the fusing process $\left(b_{2} b_{1}\right) \rightarrow b_{1}$, has a simple pole at $\zeta=i \theta_{b_{1} b_{2}}^{b_{1}}$, where

$$
\theta_{b_{1} b_{2}}^{b_{1}}:=\theta_{\left(b_{1} b_{2}\right)}^{b_{1}}+\theta_{\left(b_{2} b_{1}\right)}^{b_{1}}
$$

\footnotetext{
${ }^{3}$ In the form factor programme [BFKZ99], for a given $0<\nu<1$, there are $K$ breathers, where $K$ is the largest integer such that $K \nu<1$. Especially, if $\frac{1}{2}<\nu<1$, there is only one breather $b_{1}$, differently from our case (we are indeed not considering the Thirring model).
} 
and the same holds for the S-matrix component $S_{b_{2} b_{1}}(\zeta)$. We note that these fusing rules for the angles follow the mass parallelogram depicted in [CT16, Fig. 1]. In our construction, the poles in the component $S_{b_{2} b_{2}}(\zeta)$ do not matter. We will indeed introduce the additional concept of elementary particle in analogy with [CT16], and we assume the so-called "maximal analyticity" only for the elementary particle $b_{1}$.

These angles correspond to $s$-channel poles and in the model under investigation they are explicitly given in Table 1 . The S-matrix components $S_{b_{1} b_{1}}, S_{b_{1} b_{2}}, S_{b_{2} b_{1}}$ and $S_{b_{2} b_{2}}$ are meromorphic functions on $\mathbb{C}$, which we present below. In addition, we will introduce the matrix elements $\eta_{b_{1} b_{1}}^{b_{2}}, \eta_{b_{2} b_{1}}^{b_{1}}$ and $\eta_{b_{1} b_{2}}^{b_{1}}$ (there is no corresponding matrix element for $\left(b_{2} b_{2}\right)$, as this is not a fusion.) In a general non-diagonal case, they formally diagonalize the Smatrix components above at the corresponding pole, and their eigenvalues correspond to the residues. They were also defined in [BFKZ99] and more explicitly in [Que99, before Eq.(1.13)] (see also [BCDS90, Section 4.1.2] for models having the "breather sector") and here we adopt a slightly modified convention, as below.

\subsection{Scattering data}

The input which specifies the S-matrix of our model is the following.

- The coupling constant $\nu$, which is a parameter such that $\frac{2}{3}<\nu<\frac{4}{5}$ and the mass parameter $m>0$ which determines the masses of the breathers (see below). For the value of $\nu$ in the range above, we consider two breathers, $b_{1}, b_{2}$. Indeed, $K=2$ is the largest integer such that $K \nu<2$.

- The S-matrix components: $S_{b_{k} b_{\ell}}(\zeta)=S_{b_{k} b_{\ell}}^{\mathrm{SG}}(\zeta) S_{b_{k} b_{\ell}}^{\mathrm{CDD}}(\zeta)$, where

$$
\begin{aligned}
S_{b_{1} b_{1}}^{\mathrm{SG}}(\zeta) & =\frac{\tan \frac{1}{2 i}(\zeta+i \pi \nu)}{\tan \frac{1}{2 i}(\zeta-i \pi \nu)}, \\
S_{b_{1} b_{2}}^{\mathrm{SG}}(\zeta)=S_{b_{2} b_{1}}^{\mathrm{SG}}(\zeta) & =\frac{\tan \frac{1}{2 i}\left(\zeta+\frac{3 i \pi \nu}{2}\right)}{\tan \frac{1}{2 i}\left(\zeta-\frac{3 i \pi \nu}{2}\right)} \frac{\tan \frac{1}{2 i}\left(\zeta+\frac{i \pi \nu}{2}\right)}{\tan \frac{1}{2 i}\left(\zeta-\frac{i \pi \nu}{2}\right)}, \\
S_{b_{2} b_{2}}^{\mathrm{SG}}(\zeta) & =\frac{\tan \frac{1}{2 i}(\zeta+2 i \pi \nu)}{\tan \frac{1}{2 i}(\zeta-2 i \pi \nu)} \frac{\left(\tan \frac{1}{2 i}(\zeta+i \pi \nu)\right)^{2}}{\left(\tan \frac{1}{2 i}(\zeta-i \pi \nu)\right)^{2}} .
\end{aligned}
$$

are the breather-breather S-matrix components of the sine-Gordon model (see [BK02, Que99]), and $S_{b_{k} b_{\ell}}^{\mathrm{CDD}}$ are introduced as follows:

$$
\begin{aligned}
S_{b_{1} b_{1}}^{\mathrm{CDD}}(\zeta):= & \frac{\sinh \frac{1}{2}\left(\zeta-i \pi\left(\nu-\nu_{-}\right)\right)}{\sinh \frac{1}{2}\left(\zeta+i \pi\left(\nu-\nu_{-}\right)\right)} \cdot \frac{\sinh \frac{1}{2}\left(\zeta-i \pi\left(\nu+\nu_{+}\right)\right)}{\sinh \frac{1}{2}\left(\zeta+i \pi\left(\nu+\nu_{+}\right)\right)} \\
& \quad \times \frac{\sinh \frac{1}{2}\left(\zeta-i \pi\left(1-\nu+\nu_{-}\right)\right)}{\sinh \frac{1}{2}\left(\zeta+i \pi\left(1-\nu+\nu_{-}\right)\right)} \cdot \frac{\sinh \frac{1}{2}\left(\zeta-i \pi\left(1-\nu-\nu_{+}\right)\right)}{\sinh \frac{1}{2}\left(\zeta+i \pi\left(1-\nu-\nu_{+}\right)\right)},
\end{aligned}
$$


and expecting the bootstrap equation (see condition (S6) below), we also define

$$
\begin{aligned}
S_{b_{1} b_{2}}^{\mathrm{CDD}}(\zeta)= & S_{b_{2} b_{1}}^{\mathrm{CDD}}(\zeta) \\
:= & S_{b_{1} b_{1}}^{\mathrm{CDD}}\left(\zeta+i \theta_{\left(b_{1} b_{1}\right)}^{b_{2}}\right) S_{b_{1} b_{1}}^{\mathrm{CDD}}\left(\zeta-i \theta_{\left(b_{1} b_{1}\right)}^{b_{b^{2}}}\right) \\
= & \frac{\sinh \frac{1}{2}\left(\zeta-i \pi\left(\frac{3}{2} \nu-\nu_{-}\right)\right)}{\sinh \frac{1}{2}\left(\zeta+i \pi\left(\frac{1}{2} \nu-\nu_{-}\right)\right)} \cdot \frac{\sinh \frac{1}{2}\left(\zeta-i \pi\left(\frac{3}{2} \nu+\nu_{+}\right)\right)}{\sinh \frac{1}{2}\left(\zeta+i \pi\left(\frac{1}{2} \nu+\nu_{+}\right)\right)} \\
& \quad \times \frac{\sinh \frac{1}{2}\left(\zeta-i \pi\left(1-\frac{1}{2} \nu+\nu_{-}\right)\right)}{\sinh \frac{1}{2}\left(\zeta+i \pi\left(1-\frac{3}{2} \nu+\nu_{-}\right)\right)} \cdot \frac{\sinh \frac{1}{2}\left(\zeta-i \pi\left(1-\frac{1}{2} \nu-\nu_{+}\right)\right)}{\sinh \frac{1}{2}\left(\zeta+i \pi\left(1-\frac{3}{2} \nu-\nu_{+}\right)\right)} \\
& \quad \times \frac{\sinh \frac{1}{2}\left(\zeta-i \pi\left(\frac{1}{2} \nu-\nu_{-}\right)\right)}{\sinh \frac{1}{2}\left(\zeta+i \pi\left(\frac{3}{2} \nu-\nu_{-}\right)\right)} \cdot \frac{\sinh \frac{1}{2}\left(\zeta-i \pi\left(\frac{1}{2} \nu+\nu_{+}\right)\right)}{\sinh \frac{1}{2}\left(\zeta+i \pi\left(\frac{3}{2} \nu+\nu_{+}\right)\right)} \\
& \quad \times \frac{\sinh \frac{1}{2}\left(\zeta-i \pi\left(1-\frac{3}{2} \nu+\nu_{-}\right)\right)}{\sinh \frac{1}{2}\left(\zeta+i \pi\left(1-\frac{1}{2} \nu+\nu_{-}\right)\right)} \cdot \frac{\sinh \frac{1}{2}\left(\zeta-i \pi\left(1-\frac{3}{2} \nu-\nu_{+}\right)\right)}{\sinh \frac{1}{2}\left(\zeta+i \pi\left(1-\frac{1}{2} \nu-\nu_{+}\right)\right)}, \\
S_{b_{2} b_{2}}^{\mathrm{CDD}}(\zeta):= & S_{b_{1} b_{2}}^{\mathrm{CDD}}\left(\zeta+i \theta_{\left(b_{1} b_{1}\right)}^{b_{2}}\right) S_{b_{1} b_{2}}^{\mathrm{CDD}}\left(\zeta-i \theta_{\left(b_{1} b_{1}\right)}^{b_{2}}\right) \\
= & S_{b_{1} b_{1}}^{\mathrm{CDD}}\left(\zeta+i \theta_{b_{1} b_{1}}^{b_{2}}\right) S_{b_{1} b_{1}}^{\mathrm{CDD}}(\zeta)^{2} S_{b_{1} b_{1}}^{\mathrm{CDD}}\left(\zeta-i \theta_{b_{1} b_{1}}^{b_{2}}\right) .
\end{aligned}
$$

We do not need an explicit expression for $S_{b_{2} b_{2}}^{\mathrm{CDD}}$, and we omit computing it. $\nu_{-}$and $\nu_{+}$ are parameters satisfying the following set of conditions:

(i) $\nu_{-}, \nu_{+}>0$.

(ii) $\nu_{-} \in\left(\frac{3}{2} \nu-1, \frac{1}{2} \nu\right)$.

(iii) $\nu_{+} \in(0,1-\nu)$.

(iv) $1-\nu=\nu_{-}+\nu_{+}$.

For $\frac{2}{3}<\nu<\frac{4}{5}$, there are such $\nu_{-}, \nu_{+}$. Indeed, by rewriting every condition (i)-(iii) only in terms of $\nu_{+}$through (iv) which is equivalent to $\nu_{-}=1-\nu-\nu_{+}$, we obtain $0<\nu_{+}<1-\nu$ and $1-\frac{3}{2} \nu<\nu_{+}<2-\frac{5}{2} \nu$, which have always a nontrivial intersection for $\frac{2}{3}<\nu<\frac{4}{5}$ (on the other hand, in the interval $\frac{4}{5}<\nu<1$ there is no such intersection).

Let us take such $\nu_{-}, \nu_{+}$. (iv) is equivalent to $-\left(\frac{1}{2} \nu-\nu_{-}\right)=1-\frac{3}{2} \nu-\nu_{+}$, therefore, from $(2)$ and $\sinh \frac{1}{2}(\zeta+2 \pi i)=-\sinh \frac{1}{2} \zeta$ we have

$$
\begin{aligned}
S_{b_{1} b_{2}}^{\mathrm{CDD}}(\zeta)= & S_{b_{2} b_{1}}^{\mathrm{CDD}}(\zeta) \\
= & \frac{\sinh \frac{1}{2}\left(\zeta-i \pi\left(\frac{3}{2} \nu-\nu_{-}\right)\right)}{\sinh \frac{1}{2}\left(\zeta+i \pi\left(\frac{1}{2} \nu-\nu_{-}\right)\right)} \cdot \frac{\sinh \frac{1}{2}\left(\zeta-i \pi\left(\frac{3}{2} \nu+\nu_{+}\right)\right)}{\sinh \frac{1}{2}\left(\zeta+i \pi\left(\frac{1}{2} \nu+\nu_{+}\right)\right)} \\
& \times \frac{-1}{\sinh \frac{1}{2}\left(\zeta+i \pi\left(1-\frac{3}{2} \nu+\nu_{-}\right)\right)} \cdot \frac{\sinh \frac{1}{2}\left(\zeta-i \pi\left(1-\frac{1}{2} \nu-\nu_{+}\right)\right)}{1} \\
& \times \frac{1}{\sinh \frac{1}{2}\left(\zeta+i \pi\left(\frac{3}{2} \nu-\nu_{-}\right)\right)} \cdot \frac{\sinh \frac{1}{2}\left(\zeta-i \pi\left(\frac{1}{2} \nu+\nu_{+}\right)\right)}{1} \\
& \times \frac{\sinh \frac{1}{2}\left(\zeta-i \pi\left(1-\frac{3}{2} \nu+\nu_{-}\right)\right)}{\sinh \frac{1}{2}\left(\zeta+i \pi\left(1-\frac{1}{2} \nu+\nu_{-}\right)\right)} \cdot \frac{\sinh \frac{1}{2}\left(\zeta-i \pi\left(1-\frac{3}{2} \nu-\nu_{+}\right)\right)}{\sinh \frac{1}{2}\left(\zeta+i \pi\left(1-\frac{1}{2} \nu-\nu_{+}\right)\right)},
\end{aligned}
$$




\begin{tabular}{|c|c|c|}
\hline processes & rapidities of particles & fusion angles \\
\hline$\left(b_{1} b_{1}\right) \longrightarrow b_{2}$ & $\theta_{\left(b_{1} b_{1}\right)}^{b_{2}}=\frac{\pi \nu}{2}$ & $\theta_{b_{1} b_{1}}^{b_{2}}=\pi \nu$ \\
\hline$\left(b_{2} b_{1}\right) \longrightarrow b_{1},\left(b_{1} b_{2}\right) \longrightarrow b_{1}$ & $\theta_{\left(b_{1} b_{2}\right)}^{b_{1}}=\pi(1-\nu), \theta_{\left(b_{2} b_{1}\right)}^{b_{1}}=\frac{\pi \nu}{2}$ & $\theta_{b_{2} b_{1}}^{b_{1}}=\theta_{b_{1} b_{2}}^{b_{1}}=\pi\left(1-\frac{\nu}{2}\right)$ \\
\hline$\left(b_{2} b_{2}\right)$ not a fusion & & \\
\hline
\end{tabular}

Table 1: Fusions and angles

and it is straightforward to see that these S-matrix components have no pole in the physical strip $0<\operatorname{Im} \zeta<\pi$.

- There are only three possible fusing processes $\left(b_{1} b_{1}\right) \rightarrow b_{2},\left(b_{2} b_{1}\right) \rightarrow b_{1}$ and $\left(b_{1} b_{2}\right) \rightarrow b_{1}$. Note that $\left(b_{2} b_{2}\right)$ is not a fusion. The corresponding rapidities of particles $\theta_{\left(b_{1} b_{1}\right)}^{b_{2}}, \theta_{\left(b_{1} b_{2}\right)}^{b_{1}}$ and $\theta_{\left(b_{2} b_{1}\right)}^{b_{1}}$ are presented in the fusion table (Table 1$)$. We define the fusion angles by $\theta_{\alpha \beta}^{\gamma}:=\theta_{(\alpha \beta)}^{\gamma}+\theta_{(\beta \alpha)}^{\gamma}$ if $(\alpha \beta) \rightarrow \gamma$ is a two fusing process, where $\alpha, \beta, \gamma=b_{1}$ or $b_{2}$.

The data collected above satisfy the following properties (in general, these properties involve the charge conjugation, but for breathers it is trivial, $\bar{b}_{1}=b_{1}$ and $\bar{b}_{2}=b_{2}$ ). In the following, $k, \ell=1,2$.

(S1) Meromorphy. The functions $S_{b_{k} b_{\ell}}(\zeta)$ are meromorphic on $\mathbb{C}$.

(S2) Parity symmetry. $S_{b_{k} b_{\ell}}(\zeta)=S_{b_{\ell} b_{k}}(\zeta)$.

(S3) Unitarity. $S_{b_{k} b_{\ell}}(\zeta)^{-1}=\overline{S_{b_{\ell} b_{k}}(\bar{\zeta})}$.

(S4) Hermitian analyticity. $S_{b_{k} b_{\ell}}(\zeta)=S_{b_{k} b_{\ell}}(-\zeta)^{-1}$.

(S5) Crossing symmetry. $S_{b_{k} b_{\ell}}(i \pi-\zeta)=S_{b_{\ell} b_{k}}(\zeta)$.

(S6) Bootstrap equation. Let $\alpha, \beta, \gamma, \mu=b_{1}$ or $b_{2}$. If $(\alpha \beta) \rightarrow \gamma$ is a fusing process in Table 1, there holds

$$
S_{\mu \gamma}(\zeta)=S_{\mu \alpha}\left(\zeta+i \theta_{(\alpha \beta)}^{\gamma}\right) S_{\mu \beta}\left(\zeta-i \theta_{(\beta \alpha)}^{\gamma}\right)
$$

(S7) Value at zero. $S_{b_{k} b_{k}}(0)=-1$.

(S8) Regularity. The components $S_{b_{k} b_{\ell}}$ have only finitely many zeros in the physical strip and there is $\kappa>0$ such that $\|S\|_{\kappa}:=\sup \left\{\left|S_{b_{k} b_{\ell}}(\zeta)\right|: \zeta \in \mathbb{R}+i(-\kappa, \kappa)\right\}<\infty$ (the value of $\kappa$ depends on the parameters $\nu, \nu_{-}, \nu_{+}$).

(S9) Maximal analyticity (for $\left.b_{1}\right) \cdot{ }^{4}$ The component $S_{b_{1} b_{1}}(\zeta)$ has only two simple poles in the physical strip. They are at $i \theta_{b_{1} b_{1}}^{b_{2}}=i \pi \nu$ (called $s$-channel pole) and $i \theta_{b_{1} b_{1}}^{\prime b_{2}}:=i \pi-i \theta_{b_{1} b_{1}}^{b_{2}}=i \pi(1-\nu)$ (called $t$-channel pole, whose existence follows from crossing symmetry). Similarly, the component $S_{b_{1} b_{2}}(\zeta)$ has also only two simple poles, i.e. an $s$-channel pole at $i \theta_{b_{1} b_{2}}^{b_{1}}=i \pi\left(1-\frac{\nu}{2}\right)$ and a $t$-channel pole at $i \theta_{b_{1} b_{2}}^{\prime b_{1}}:=i \pi-i \theta_{b_{2} b_{1}}^{b_{1}}=\frac{i \pi \nu}{2}$.

\footnotetext{
${ }^{4}$ We call this "maximal analyticity" because each $s$-channel pole at $i \theta_{b_{1} b_{k}}^{b_{\ell}}$ has a corresponding entry $\left(b_{1} b_{k}\right) \rightarrow b_{\ell}$ in the fusion Table 1 . It should be noted that this is required only for the S-matrix components containing $b_{1}$, the "elementary particle" defined below.
} 
(S10) No higher poles. $S_{b_{1} b_{k}}$ have no double or higher poles in the physical strip, $k=1,2$.

(S11) Positive residue (for $\left.b_{1}\right)$. If $\left(b_{1} b_{k}\right) \rightarrow b_{\ell}$ is a fusing process, then

$$
R_{b_{1} b_{k}}^{b_{\ell}}:=\operatorname{Res}_{\zeta=i \theta_{b_{1} b_{k}}^{b_{\ell}}} S_{b_{1} b_{k}}(\zeta) \in i \mathbb{R}_{+}
$$

We note that while some of these properties are well known in the form factor programme, see e.g. [BFKZ99, Sec. 2] and [BCDS90, Section 4], for the proof of weak-wedge commutativity we additionally require properties (S11) (see proof of Theorem 5.1 below) which is known for many models (e.g. [BCDS90, Section 4.1]), and (S7) (relevant for the relations among the residues and their signs, see proof of (S11) and comments before Eq. (4)) and the absence of double or higher order poles of the S-matrix components $S_{b_{1} b_{k}}, k=1,2$, in the physical strip as mentioned above.

We are currently not able to treat directly observables generating particles $b_{k}, k \neq$ 1 from the vacuum. What we do is to try to construct observables generating the particle $b_{k}$ from the vacuum and hope that they generate the whole Hilbert space. The assumption (S10) or an analogous assumptions exclude some S-matrices in the literature, e.g. see [BCDS90, Section 4.2.4].

\section{Proof of the properties.}

- (S1)-(S6) and (S8). These properties are already satisfied by the S-matrix with components $S_{\mathrm{SG}_{k} b_{\ell}}$ of the sine-Gordon model (and well-known in the literature). It is also straightforward to check that $S_{b_{k} b_{\ell}}^{\mathrm{CDD}}(\zeta)$ satisfy (S1)-(S5) and (S8). As for (S6), we have by construction

$$
S_{b_{1} b_{2}}^{\mathrm{CDD}}(\zeta)=S_{b_{1} b_{1}}^{\mathrm{CDD}}\left(\zeta+\frac{i \pi \nu}{2}\right) S_{b_{1} b_{1}}^{\mathrm{CDD}}\left(\zeta-\frac{i \pi \nu}{2}\right)
$$

By the properties mentioned above (in particular, hermitian analyticity), we have

$$
S_{b_{1} b_{1}}^{\mathrm{CDD}}(\zeta)=S_{b_{1} b_{2}}^{\mathrm{CDD}}\left(\zeta+\frac{i \pi \nu}{2}\right) S_{b_{1} b_{1}}^{\mathrm{CDD}}(\zeta-i \pi(1-\nu))
$$

Similarly, the bootstrap for $b_{2}$ can be satisfied by construction.

Therefore, the products $S_{b_{k} b_{\ell}}(\zeta)=S_{b_{k} b_{\ell}}^{\mathrm{SG}}(\zeta) S_{b_{k} b_{\ell}}^{\mathrm{CDD}}(\zeta)$ satisfy them as well.

- (S7). It is easy to see that $S_{b_{k} b_{k}}^{\mathrm{SG}}(0)=-1$, while $S_{b_{k} b_{k}}^{\mathrm{CDD}}(0)=1$, therefore, we have $S_{b_{k} b_{k}}(0)=-1$.

- (S9). The expression of $S_{b_{1} b_{k}}^{\mathrm{CDD}}(\zeta)$ does not have poles in the physical strip, so the pole structure of $S_{b_{1} b_{k}}(\zeta)$ is determined by $S_{b_{1} b_{k}}^{\mathrm{SG}}(\zeta)$, which is easy to check (and known in the literature).

- (S10) This is clear from the explicit expressions of $S_{b_{1} b_{k}}^{\mathrm{CDD}}(\zeta)$ and $S_{b_{1} b_{k}}^{\mathrm{SG}}(\zeta)$. 
- (S11) is violated in the sine-Gordon model, indeed

$$
R_{b_{1} b_{1}}^{\mathrm{SG} b_{2}}:=\operatorname{Res}_{\zeta=i b_{b_{1} b_{1}}^{b_{2}}} S_{b_{1} b_{1}}^{\mathrm{SG}}(\zeta)=2 i \tan (\pi \nu) \in-i \mathbb{R}_{+},
$$

for our range of $\nu \in\left(\frac{2}{3}, \frac{4}{5}\right)$.

On the other hand, by counting the zeros on the imaginary line and by recalling that $S_{b_{1} b_{1}}^{\mathrm{CDD}}(0)=1$, we see that $S_{b_{1} b_{1}}^{\mathrm{CDD}}(i \pi \nu)<0$, hence we obtain $R_{b_{1} b_{1}}^{b_{2}}=\operatorname{Res}_{\zeta=i \pi \nu} S_{b_{1} b_{1}}(\zeta) \in$ $i \mathbb{R}_{+}$as desired. From this it follows that $R_{b_{1} b_{2}}^{b_{1}} \in i \mathbb{R}_{+}$as well, since we will see below that $R_{b_{1} b_{2}}^{b_{1}}=R_{b_{1} b_{1}}^{b_{2}}$.

Remark 2.1. If $\nu<\frac{2}{3}$, then $S_{b_{1} b_{2}}$ has one more pair of simple poles and violates (S9). If $\frac{4}{5}<\nu$, combined with (ii) and (iv) $\left(1-\frac{3}{2} \nu<\nu_{+}<2-\frac{5}{2} \nu\right)$, it forces that $\nu_{+}<0$ and the condition (S11) fails.

The residues of $S_{b_{1} b_{k}}(\zeta)$ will play an important role, so we give them symbols.

$$
\begin{aligned}
& R_{b_{1} b_{k}}^{b_{\ell}}:=\operatorname{Res}_{\zeta=i \theta_{b_{1} b_{k}}^{b_{\ell}}} S_{b_{1} b_{k}}(\zeta), \quad R_{b_{1} b_{k}}^{b_{\ell}}:=\operatorname{Res}_{\zeta=i \theta_{b_{1} b_{k}}^{\prime b_{\ell}}} S_{b_{1} b_{k}}(\zeta) \\
& R_{b_{k} b_{1}}^{b_{\ell}}:=\operatorname{Res}_{\zeta=i \theta_{b_{k} b_{1}}^{b_{\ell}}} S_{b_{k} b_{1}}(\zeta), \quad R_{b_{k} b_{1}}^{b_{\ell}}:=\operatorname{Res}_{\zeta=i \theta_{b_{k} b_{1}}^{\prime b_{\ell}}} S_{b_{k} b_{1}}(\zeta)
\end{aligned}
$$

and it follows that $R_{b_{1} b_{k}}^{b_{\ell}}=R_{b_{k} b_{1}}^{b_{\ell}}$.

As before, we also introduce ${ }^{5}$ the symbols $\eta_{b_{1} b_{1}}^{b_{2}}$ and $\eta_{b_{2} b_{1}}^{b_{1}}$ by the following formula:

$$
\eta_{b_{1} b_{1}}^{b_{2}}=i \sqrt{2 \pi\left|R_{b_{1} b_{1}}^{b_{2}}\right|}, \quad \eta_{b_{1} b_{2}}^{b_{1}}=i \sqrt{2 \pi\left|R_{b_{1} b_{2}}^{b_{1}}\right|}, \quad \eta_{b_{2} b_{1}}^{b_{1}}=i \sqrt{2 \pi\left|R_{b_{2} b_{1}}^{b_{1}}\right|} .
$$

Furthermore, by convention, we set to zero any residues and matrix elements of the above type which do not correspond to a fusion in Table 1. From the properties (S2)-(S7) of the S-matrix, there is a number of other properties of the fusion angles and of the residues that follow, and we refer for the proofs to [CT16, Sec. 2.1]. We would mention here only the following. The residue of the $t$-channel pole is related to the residue of the s-channel pole by $R_{b_{1} b_{1}}^{b_{2}}=-R_{b_{1} b_{1}}^{b_{2}}$ and $R_{b_{2} b_{1}}^{b_{1}}=-R_{b_{1} b_{2}}^{b_{1}}$, and that by (S2), $R_{b_{1} b_{2}}^{b_{1}}=R_{b_{2} b_{1}}^{b_{1}}$. (S6) and (S7) imply that $R_{b_{1} b_{1}}^{b_{2}}=R_{b_{1} b_{2}}^{b_{1}}$. Furthermore, if $\left(b_{1} b_{k}\right) \rightarrow b_{\ell}$ is a fusing process, the fusion angles are also related by

$$
\pi-\theta_{b_{1} b_{k}}^{b_{\ell}}=\theta_{\left(b_{1} b_{\ell}\right)}^{b_{k}}, \quad \theta_{\left(b_{\ell} b_{1}\right)}^{b_{k}}=\theta_{\left(b_{k} b_{1}\right)}^{b_{\ell}} .
$$

From the equality $R_{b_{1} b_{1}}^{b_{2}}=R_{b_{1} b_{2}}^{b_{1}}$ and the parity $R_{b_{1} b_{2}}^{b_{1}}=R_{b_{2} b_{1}}^{b_{1}}$, it also holds that $\eta_{b_{1} b_{1}}^{b_{2}}=$ $\eta_{b_{1} b_{2}}^{b_{1}}=\eta_{b_{2} b_{1}}^{b_{1}}$

Particle spectrum. Given the mass parameter $m>0$, we define the masses of the particles as

$$
m_{b_{1}}=2 m \sin \frac{\nu \pi}{2}, \quad m_{b_{2}}=2 m \sin \frac{2 \nu \pi}{2} .
$$

\footnotetext{
${ }^{5}$ We use a slightly different convention from [Que99]: For a fusing process $(\alpha \beta) \rightarrow \gamma$, we have $\eta_{\alpha \beta}^{\gamma}=$ $\sqrt{2 \pi} \eta_{\alpha \beta}^{\gamma(\text { Quella) }}$.
} 
They satisfy the following "fusion" rule:

$$
m_{b_{2}}=m_{b_{1}} \cos \theta_{\left(b_{1} b_{1}\right)}^{b_{2}}+m_{b_{1}} \cos \theta_{\left(b_{1} b_{1}\right)}^{b_{2}}, \quad m_{b_{1}}=m_{b_{1}} \cos \theta_{\left(b_{1} b_{2}\right)}^{b_{1}}+m_{b_{2}} \cos \theta_{\left(b_{2} b_{1}\right)}^{b_{1}},
$$

which are a particular case of [CT16, Eq. (1)].

As $b_{1}$ plays a special role in our methods, we call it an elementary particle as in [CT16, Sec. 2.1].

\section{The physical Hilbert space}

From the scattering data of Section 2.2, we construct basic mathematical structures for the wedge-observables in the quantum field theory on the $S$-symmetric Fock space. The construction can be thought of as a kind of deformation of a free field theory with the input given by the S-matrix. The single-particle Hilbert space accommodates the two species of particles:

$$
\mathcal{H}_{1}=\bigoplus_{k=1,2} \mathcal{H}_{1, b_{k}}, \quad \mathcal{H}_{1, b_{k}}=L^{2}(\mathbb{R}, d \theta) .
$$

An element $\Psi_{1} \in \mathcal{H}_{1}$ can be identified as a vector valued function with components $\theta \mapsto$ $\Psi_{1}^{b_{k}}(\theta)$. On the unsymmetrized $n$-particle space $\mathcal{H}_{1}^{\otimes n}$, there is a unitary representation $D_{n}$ of the symmetric group $\mathfrak{G}_{n}$ which, with $\boldsymbol{\theta}:=\left(\theta_{1}, \cdots, \theta_{n}\right)$, acts as

$$
\left(D_{n}\left(\tau_{j}\right) \Psi_{n}\right)^{\boldsymbol{b}_{\boldsymbol{k}}}(\boldsymbol{\theta})=S_{b_{k_{j+1}} b_{k_{j}}}\left(\theta_{j+1}-\theta_{j}\right) \Psi_{n}^{b_{k_{1}} \cdots b_{k_{j+1}} b_{k_{j}} \cdots b_{k_{n}}}\left(\theta_{1}, \cdots, \theta_{j+1}, \theta_{j}, \cdots, \theta_{n}\right),
$$

where $k_{1}, \ldots, k_{n} \in\{1,2\}, \boldsymbol{\theta}:=\left(\theta_{1}, \ldots, \theta_{n}\right), \boldsymbol{b}_{\boldsymbol{k}}:=\left(b_{k_{1}}, \ldots, b_{k_{n}}\right)$ and $\tau_{j} \in \mathfrak{G}_{n}$ is the transposition $(j, j+1) \rightarrow(j+1, j)$.

The full Hilbert space $\mathcal{H}$ is $\mathcal{H}:=\bigoplus_{n=0}^{\infty} \mathcal{H}_{n}$ with $\mathcal{H}_{0}=\mathbb{C} \Omega$, where $\mathcal{H}_{n}=P_{n} \mathcal{H}_{1}^{\otimes n}$ and $P_{n}:=\frac{1}{n !} \sum_{\sigma \in \mathfrak{G}_{n}} D_{n}(\sigma)$ is an orthogonal projection. The elements of $\mathcal{H}$ are $L^{2}$-sequences $\Psi=\left(\Psi_{0}, \Psi_{1}, \ldots\right)$, where $\Psi_{n}$ are $S$-symmetric functions, namely invariant under the action of $\mathfrak{G}_{n}$. Finally, we denote by $\mathcal{D}$ the linear hull (without closure) of $\left\{\mathcal{H}_{n}\right\}$.

There is a unitary representation $U$ of the proper orthochronous Poincaré group $\mathcal{P}_{+}^{\uparrow}$ on $\mathcal{H}$ which preserves each $\mathcal{H}_{n}$,

$$
U:=\bigoplus_{n} U_{n}, \quad(U(a, \lambda) \Psi)_{n}^{\boldsymbol{b}_{\boldsymbol{k}}}(\boldsymbol{\theta}):=\exp \left(i \sum_{l=1}^{n} p_{b_{k_{l}}}\left(\theta_{l}\right) \cdot a\right) \Psi_{n}^{\boldsymbol{b}_{\boldsymbol{k}}}\left(\theta_{1}-\lambda, \cdots, \theta_{n}-\lambda\right),
$$

where $p_{b_{k_{l}}}(\theta)=\left(m_{b_{k_{l}}} \cosh \theta, m_{b_{k_{l}}} \sinh \theta\right)$. Additionally, there is an antiunitary representation of the CPT operator on $\mathcal{H}$ :

$$
J:=\bigoplus_{n} J_{n}, \quad(J \Psi)_{n}^{\boldsymbol{b}_{\boldsymbol{k}}}(\boldsymbol{\theta}):=\overline{\Psi_{n}^{b_{k_{n}} \ldots b_{k_{1}}}\left(\theta_{n}, \ldots, \theta_{1}\right)}
$$

We consider test functions with multi-components whose components are chosen as $g \in$ $\bigoplus_{k=1}^{2} \mathscr{S}\left(\mathbb{R}^{2}\right)$ with $g_{b_{k}} \in \mathscr{S}\left(\mathbb{R}^{2}\right)$, and we adopt the following convention:

$$
g_{b_{k}}^{ \pm}(\theta):=\frac{1}{2 \pi} \int d^{2} x g_{b_{k}}(x) e^{ \pm i p_{b_{k}}(\theta) \cdot x} .
$$


We note that ${ }^{6}$ if $g_{b_{k}}$ is supported in $W_{\mathrm{R}}$, then $g_{b_{k}}^{+}(\theta)$ has a bounded analytic continuation in $\mathbb{R}+i(-\pi, 0)$ and $\left|g_{b_{k}}^{+}(\theta+i \lambda)\right|$ decays rapidly as $\theta \rightarrow \pm \infty$ in the strip for $\lambda \in(-\pi, 0)$. Moreover, $g_{b_{k}}^{+}(\theta-i \pi)=g_{b_{k}}^{-}(\theta)$.

There is a natural action of the proper Poincaré group on $\mathbb{R}^{2}$ and on the space of test functions, denoted by $g_{(a, \lambda)}$, and it is compatible with the action on the one-particle space:

$$
\left(g_{(a, \lambda)}\right)_{b_{k}}^{ \pm}=U_{1}(a, \lambda) g_{b_{k}}^{ \pm} .
$$

The CPT transformation acts also on multi-components test functions, which we denote by $j$, as $g \mapsto g_{j},\left(g_{j}\right)_{b_{k}}(x):=\overline{g_{b_{k}}(-x)}$, and this is again compatible with $J_{1}:\left(g_{j}\right)_{b_{k}}^{ \pm}(\theta)=$ $J_{1} g_{b_{k}}^{ \pm}(\theta)=\overline{g_{b_{k}}^{ \pm}(\theta)}$.

Moreover, we introduce the complex conjugate of a multi-component test function by $\left(g^{*}\right)_{b_{k}}(x):=\overline{g_{b_{k}}(x)}$ and if $g=g^{*}$, then we say that $g$ is real and it follows that $\overline{g_{b_{k}}^{ \pm}(\bar{\zeta})}=g_{b_{k}}^{\mp}(\zeta)$ (c.f. [LS14, Proposition 3.1]).

\section{Zamolodchikov-Faddeev algebra}

Similarly to [LS14], creators and annihilators $z_{b_{k}}^{\dagger}(\theta), z_{b_{k}}(\theta)$ are introduced in the $S$-symmetric Fock space $\mathcal{H}$. For $\varphi \in \mathcal{H}_{1}$, their actions on vectors $\Psi=\left(\Psi_{n}\right) \in \mathcal{D}$ are given by

$$
\begin{aligned}
(z(\varphi) \Psi)_{n}^{\boldsymbol{b}_{\boldsymbol{k}}}(\boldsymbol{\theta}) & =\sqrt{n+1} \sum_{l=1,2} \int d \theta^{\prime} \overline{\varphi^{b_{l}}\left(\theta^{\prime}\right)} \Psi_{n+1}^{b_{b} \boldsymbol{b}_{\boldsymbol{k}}}\left(\theta^{\prime}, \boldsymbol{\theta}\right), \\
z^{\dagger}(\varphi) & =(z(\varphi))^{*}
\end{aligned}
$$

(see [LS14, Proposition 2.4]) and they formally fulfill the following Zamolodchikov-Faddeev algebra:

$$
\begin{aligned}
& z_{b_{k}}^{\dagger}(\theta) z_{b_{l}}^{\dagger}\left(\theta^{\prime}\right)=S_{b_{k} b_{l}}\left(\theta-\theta^{\prime}\right) z_{b_{l}}^{\dagger}\left(\theta^{\prime}\right) z_{b_{k}}^{\dagger}(\theta), \\
& z_{b_{k}}(\theta) z_{b_{l}}\left(\theta^{\prime}\right)=S_{b_{k} b_{l}}\left(\theta-\theta^{\prime}\right) z_{b_{l}}\left(\theta^{\prime}\right) z_{b_{k}}(\theta), \\
& z_{b_{k}}(\theta) z_{b_{l}}^{\dagger}\left(\theta^{\prime}\right)=S_{b_{l} b_{k}}\left(\theta^{\prime}-\theta\right) z_{b_{l}}^{\dagger}\left(\theta^{\prime}\right) z_{b_{k}}(\theta)+\delta^{b_{k} b_{l}} \delta\left(\theta-\theta^{\prime}\right) \mathbb{1}_{\mathcal{H}} .
\end{aligned}
$$

They are opereator-valued distributions defined on $\mathcal{D}$ and bounded on each $n$-particle space $\mathcal{H}_{n}$ when smeared by a test function.

Let $f \in \bigoplus_{k=1,2} \mathscr{S}\left(\mathbb{R}^{2}\right)$, we define

$$
\begin{aligned}
\phi(f) & :=z^{\dagger}\left(f^{+}\right)+z\left(J_{1} f^{-}\right) \\
& \left(=\sum_{k=1,2} \int d \theta\left(f_{b_{k}}^{+}(\theta) z_{b_{k}}^{\dagger}(\theta)+\left(J_{1} f^{-}\right)_{b_{k}}(\theta) z_{b_{k}}(\theta)\right)\right) .
\end{aligned}
$$

This multi-component quantum field ${ }^{7}$ is defined on the subspace $\mathcal{D}$ of $\mathcal{H}$ of vectors with finite particle number and the properties listed in [LS14, Proposition 3.1] are fulfilled, as long as

\footnotetext{
${ }^{6}$ Our convention of the Lorentz metric is $a \cdot b=a_{0} b_{0}-a_{1} b_{1}$.

${ }^{7}$ If the S-matrix $S(\zeta)$ were analytic in the physical strip, $\phi(f)$ could be considered as an observable localized in the standard left wedge $W_{\mathrm{L}}$ and if furthermore $S$ is diagonal with additional regularity conditions, one would be able to obtain a Haag-Kastler net with minimal length [LS14, AL16]. In contrast, our S-matrix has poles in the physical strip.
} 
the analyticity in the physical strip is not used. We also introduce $\phi^{\prime}$, the reflected field defined for $g \in \mathscr{S}\left(\mathbb{R}^{2}\right)$,

$$
\phi^{\prime}(g):=J \phi\left(g_{j}\right) J=z^{\prime \dagger}\left(g^{+}\right)+z^{\prime}\left(J_{1} g^{-}\right)
$$

where $z^{\prime}, z^{\prime \dagger}$ are the reflected creators and annihilators $z_{b_{k}}^{\prime}(\theta):=J z_{b_{k}}(\theta) J$ and $z_{b_{k}}^{\prime \dagger}(\theta):=$ $J z_{b_{k}}^{\dagger}(\theta) J$.

For the class of two-particle S-matrices $S(\theta)$ with components which are not analytic in the physical strip $\theta \in \mathbb{R}+i(0, \pi)$, we have $\left[\phi(f), \phi^{\prime}(g)\right] \neq 0$, namely, even weak commutativity fails for $\phi, \phi^{\prime}$. The goal of the present paper is to find alternative wedge-observables for the S-matrix of the sine-Gordon model.

\section{The bound state operator}

We introduce an operator $\chi(f)$ similarly to [CT16], which we again call the "bound state operator". Its mathematical structure corresponds to our fusion table, which is same as the breather-breather fusing processes in the sine-Gordon model with two breathers. In this model, the "elementary particle" is $b_{1}$, and we restrict ourselves to the case where $f_{b_{1}}$ is the only non-zero component of a test function $f$.

\subsection{Definitions and domains}

We define $\chi(f)$ as an unbounded operator on the $S$-symmetric Fock space $\mathcal{H}$. Recall that for $s<t, H^{2}\left(\mathbb{S}_{s, t}\right)$ is the Hardy space of analytic functions $\Psi$ in $\mathbb{S}_{s, t}:=\mathbb{R}+i(s, t)$ such that $\Psi(\theta+i \lambda)$ is $L^{2}(\mathbb{R})$ as a function of $\theta$ for each $\lambda \in(s, t)$ and their $L^{2}$-norm is uniformly bounded for $\lambda$. For a multi-component test function $f$ whose only non-zero component is $f_{b_{1}}$ and is supported in $W_{\mathrm{L}}$, its action on $\mathcal{H}_{1}$ is given as follows:

$$
\begin{aligned}
& \operatorname{Dom}\left(\chi_{1}(f)\right):=H^{2}\left(\mathbb{S}_{-\theta_{\left(b_{1} b_{1}\right)}^{b_{2}}, 0}\right) \oplus H^{2}\left(\mathbb{S}_{-\theta_{\left(b_{2} b_{1}\right)}^{b_{1}}, 0}\right) \\
& \left(\chi_{1}(f) \xi\right)_{b_{k}}(\theta):= \begin{cases}-i \eta_{b_{1} b_{2}}^{b_{1}} f_{b_{1}}^{+}\left(\theta+i \theta_{\left(b_{1} b_{2}\right)}^{b_{1}}\right) \xi_{b_{2}}\left(\theta-i \theta_{\left(b_{2} b_{1}\right)}^{b_{1}}\right) & \text { if } k=1, \\
-i \eta_{b_{1} b_{1}}^{b_{2}} f_{b_{1}}^{+}\left(\theta+i \theta_{\left(b_{1} b_{1}\right)}^{b_{2}}\right) \xi_{b_{1}}\left(\theta-i \theta_{\left(b_{1} b_{1}\right)}^{b_{2}}\right) & \text { if } k=2 .\end{cases}
\end{aligned}
$$

where $\eta_{b_{1} b_{1}}^{b_{2}}, \eta_{b_{1} b_{2}}^{b_{1}}$ are the matrix elements introduced in Sec. 2.2, see Eq. (3). Actually, $\theta_{\left(b_{2} b_{1}\right)}^{b_{1}}=\theta_{\left(b_{2} b_{1}\right)}^{b_{2}}=\frac{\pi \nu}{2}$, hence $\operatorname{Dom}\left(\chi_{1}(f)\right)=H^{2}\left(\mathbb{S}_{-\frac{\pi \nu}{2}, 0}\right)^{\oplus 2}$, but we often keep the notation above for homogeneity.

The full operator $\chi(f)$ is the direct sum of its components $\chi_{n}(f)$ on $\mathcal{H}_{n}$ :

$$
\chi(f)=\bigoplus_{n=0}^{\infty} \chi_{n}(f), \quad \chi_{n}(f):=n P_{n}\left(\chi_{1}(f) \otimes \mathbb{1} \otimes \cdots \otimes \mathbb{1}\right) P_{n} .
$$

Similarly, and as in [CT16], we introduce the reflected bound state operator $\chi^{\prime}(g)$ for a test function $g$ supported in the right wedge $W_{\mathrm{R}}$. Again, its one particle projection for $g$ having 
only one non-zero component $g_{b_{1}}$ is given by

$$
\begin{aligned}
& \operatorname{Dom}\left(\chi_{1}^{\prime}(g)\right):=H^{2}\left(\mathbb{S}_{0, \theta_{\left(b_{1} b_{1}\right)}^{b_{2}}}\right) \oplus H^{2}\left(\mathbb{S}_{0, \theta_{\left(b_{2} b_{1}\right)}^{b_{1}}}\right) \\
& \left(\chi_{1}^{\prime}(g) \xi\right)_{b_{k}}(\theta):= \begin{cases}-i \eta_{b_{1} b_{2}}^{b_{1}} g_{b_{1}}^{+}\left(\theta-i \theta_{\left(b_{1} b_{2}\right)}^{b_{1}}\right) \xi_{b_{2}}\left(\theta+i \theta_{\left(b_{2} b_{1}\right)}^{b_{1}}\right) & \text { if } k=1, \\
-i \eta_{b_{1} b_{1}}^{b_{2}} g_{b_{1}}^{+}\left(\theta-i \theta_{\left(b_{1} b_{1}\right)}^{b_{2}}\right) \xi_{b_{1}}\left(\theta+i \theta_{\left(b_{1} b_{1}\right)}^{b_{2}}\right) & \text { if } k=2 .\end{cases}
\end{aligned}
$$

The full operator on $\mathcal{H}$ is given by

$$
\chi^{\prime}(g)=\bigoplus_{n} \chi_{n}^{\prime}(g), \quad \chi_{n}^{\prime}(g):=n P_{n}\left(\mathbb{1} \otimes \cdots \otimes \mathbb{1} \otimes \chi_{1}^{\prime}(g)\right) P_{n}
$$

This operator is related to $\chi$ by the CPT operator $J$ :

$$
\chi^{\prime}(g)=J \chi\left(g_{j}\right) J
$$

To see this, let us consider the one-particle components. By recalling the expression (6),

$$
\begin{aligned}
& \left(J \chi_{1}\left(g_{j}\right) J \xi\right)_{b_{\ell}}(\theta)=\overline{\left(\chi_{1}\left(g_{j}\right) J \xi\right)_{b_{\ell}}(\theta)} \\
& =\overline{-i \eta_{b_{1} b_{k}}^{b_{\ell}}\left(g_{j}\right)_{b_{1}}^{+}\left(\theta+i \theta_{\left(b_{1} b_{k}\right)}^{b_{\ell}}\right)(J \xi)_{b_{k}}\left(\theta-i \theta_{\left(b_{k} b_{1}\right)}^{b_{\ell}}\right)} \\
& =-i \eta_{b_{1} b_{k}}^{b_{\ell}} g_{b_{1}}^{+}\left(\theta-i \theta_{\left(b_{1} b_{k}\right)}^{b_{\ell}}\right) \xi_{b_{k}}\left(\theta+i \theta_{\left(b_{k} b_{1}\right)}^{b_{\ell}}\right) \\
& =\left(\chi^{\prime}(g) \xi\right)_{b_{\ell}}(\theta) \text {, }
\end{aligned}
$$

where $l=1$ or 2 and $k=2$ or 1 , respectively, and we used that $-i \eta_{\alpha \beta}^{\gamma} \in \mathbb{R}$. As $J_{n}$ commutes with $P_{n}$, we have $\chi_{n}^{\prime}(g)=J_{n} \chi_{n}\left(g_{j}\right) J_{n}$. Since the whole operators $\chi(g)$ and $\chi^{\prime}(g)$ are defined as the direct sum, the desired equality follows.

We give some more explicit expressions of Eqs. (7) and (8) by applying them to a $n$ particle vector which we assume to be $S$-symmetric and in the domain of $\chi_{1}(f) \otimes \mathbb{1} \otimes \cdots \otimes \mathbb{1}$ and of $\mathbb{1} \otimes \cdots \otimes \mathbb{1} \otimes \chi_{1}^{\prime}(g)$, respectively. We have, from (S6), (S2) and (S4) exactly as in [CT16, Section 3.2],

$$
\begin{aligned}
& \left(\chi(f) \Psi_{n}\right)^{b_{k_{1}} \cdots b_{k_{n}}}\left(\theta_{1}, \ldots, \theta_{n}\right) \\
& =-i \sum_{\substack{1 \leq \ell \leq n, \alpha_{\ell}=b_{1}, b_{2} \\
\eta_{b_{1} \alpha_{\ell}}}}^{b_{k_{\ell}}}\left(\prod_{1 \leq j \leq \ell-1} S_{b_{1} b_{k_{j}}}\left(\theta_{\ell}-\theta_{j}+i \theta_{\left(b_{1} \alpha_{\ell}\right)}^{b_{k_{\ell}}}\right)\right) \\
& \quad \times f_{b_{1}}^{+}\left(\theta_{\ell}+i \theta_{\left(b_{1} \alpha_{\ell}\right)}^{b_{k_{\ell}}}\right) \Psi_{n}^{b_{k_{1}} \cdots b_{k_{\ell-1}} \alpha_{\ell} b_{k_{\ell+1}} \ldots b_{k_{n}}}\left(\theta_{1}, \cdots, \theta_{\ell-1}, \theta_{\ell}-i \theta_{\left(\alpha_{\ell} b_{1}\right)}^{b_{k_{\ell}}}, \theta_{\ell+1}, \cdots \theta_{n}\right),
\end{aligned}
$$

where $k_{1}, \ldots, k_{n}=1,2$ and we applied our convention that $\eta_{\alpha \beta}^{\gamma}=0$ if $(\alpha \beta) \rightarrow \gamma$ is not a fusion, and terms containing such $\eta_{\alpha \beta}^{\gamma}$ should be ignored (even if it contains expressions such as $\Psi\left(\cdots, \theta-i \theta_{(\beta \alpha)}^{\gamma}, \cdots\right)$ which can be meaningless, as it might be outside the domain of analyticity). 
We have a similar expression for $\chi^{\prime}(g)$ :

$$
\begin{aligned}
& \left(\chi^{\prime}(g) \Psi_{n}\right)^{b_{k_{1}} \ldots b_{k_{n}}}\left(\theta_{1}, \ldots, \theta_{n}\right)= \\
& \quad=-i \sum_{\substack{1 \leq \ell \leq n, \alpha_{\ell}=b_{1}, b_{2} \\
\quad}} \eta_{b_{1} \alpha_{\ell}}^{b_{k_{\ell}}}\left(\prod_{\ell+1 \leq j \leq n} S_{b_{k_{j}} b_{1}}\left(\theta_{j}-\theta_{\ell}+i \theta_{\left(b_{1} \alpha_{\ell}\right)}^{b_{k_{\ell}}}\right)\right) \\
& \quad \times g_{b_{1}}^{+}\left(\theta_{\ell}-i \theta_{\left(b_{1} \alpha_{\ell}\right)}^{b_{k_{\ell}}}\right) \Psi_{n}^{b_{k_{1}} \ldots b_{k_{\ell-1}} \alpha_{\ell} b_{k_{\ell+1}} \cdots b_{k_{n}}}\left(\theta_{1}, \cdots, \theta_{\ell-1}, \theta_{\ell}+i \theta_{\left(\alpha_{\ell} b_{1}\right)}^{b_{k_{\ell}}}, \theta_{\ell+1} \cdots \theta_{n}\right) .
\end{aligned}
$$

\subsection{Some properties}

We remark here on some of the properties of $\chi(f)$, noting that analogous properties hold by construction for $\chi^{\prime}(g)$. For a multi-component real test function $f$ whose only non-zero component is $f_{b_{1}}$ which is real, we can prove that $\chi(f)$ is densely defined and symmetric.

By construction, $\chi_{1}(f)$ is densely defined. To show that $\chi_{1}(f)$ is symmetric, we take two vectors $\xi, \psi \in \operatorname{Dom}\left(\chi_{1}(f)\right)$ whose components have compact inverse Fourier transform. We can show that these vectors form a core for $\chi_{1}(f)$. By recalling that $\eta_{b_{1} b_{k}}^{b_{\ell}}=0$ unless $k=1, \ell=2$ or $k=2, \ell=1$, we compute on vectors $\xi, \psi$ from the core:

$$
\begin{aligned}
\left\langle\psi, \chi_{1}(f) \xi\right\rangle & =-\sum_{k, \ell} i \eta_{b_{1} b_{k}}^{b_{\ell}} \int d \theta \overline{\psi^{b_{\ell}(\theta)}} f_{b_{1}}^{+}\left(\theta+i \theta_{\left(b_{1} b_{k}\right)}^{b_{\ell}}\right) \xi^{b_{k}}\left(\theta-i \theta_{\left(b_{k} b_{1}\right)}^{b_{\ell}}\right) \\
& =-\sum_{k, \ell} i \eta_{b_{1} b_{k}}^{b_{\ell}} \int d \theta \overline{f_{b_{1}}^{+}\left(\theta+i \pi-i \theta_{\left(b_{1} b_{k}\right)}^{b_{\ell}}\right.} \overline{\psi^{b_{\ell}(\theta)}} \xi^{b_{k}}\left(\theta-i \theta_{\left(b_{k} b_{1}\right)}^{b_{\ell}}\right) \\
& =-\sum_{k \ell} i \eta_{b_{1} b_{k}}^{b_{\ell}} \int d \theta \overline{f_{b_{1}}^{+}\left(\theta+i \pi-i \theta_{b_{1} b_{k}}^{b_{\ell}}\right) \psi^{b_{\ell}}\left(\theta-i \theta_{\left(b_{k} b_{1}\right)}^{b_{\ell}}\right)} \xi^{b_{k}}(\theta) \\
& =-\sum_{k \ell} i \eta_{b_{1} b_{\ell}}^{b_{k}} \int d \theta \overline{f_{b_{1}}^{+}\left(\theta+i \theta_{\left(b_{1} b_{\ell}\right)}^{b_{k}}\right) \psi^{b_{\ell}}\left(\theta-i \theta_{\left(b_{\ell} b_{1}\right)}^{b_{k}}\right)} \xi^{b_{k}}(\theta)=\left\langle\chi_{1}(f) \psi, \xi\right\rangle,
\end{aligned}
$$

where in the second equality we used the property $f^{+}(\theta+i \lambda)=\overline{f^{+}(i \pi-\theta-i \lambda)}$ explained at the end of Sec. 3. In the third equality we used the Cauchy theorem and performed the shift $\theta \rightarrow \theta+i \theta_{\left(b_{k} b_{1}\right)}^{b_{\ell}}$, since the integrand is analytic, bounded and rapidly decreasing in the strip $\mathbb{R}+i(0, \pi)$ due to $\xi, \psi$ being the Fourier transforms of compactly supported functions and the properties of $f^{+}$. In the fourth equality we used the properties $\pi-\theta_{b_{1} b_{1}}^{b_{2}}=\theta_{\left(b_{1} b_{2}\right)}^{b_{1}}$, $\theta_{\left(b_{1} b_{1}\right)}^{b_{2}}=\theta_{\left(b_{2} b_{1}\right)}^{b_{1}}$ and $\eta_{b_{1} b_{1}}^{b_{2}}=\eta_{b_{1} b_{2}}^{b_{1}}$ from Sec. 2.2 .

We can show that $\chi_{n}(f)$ is densely defined and symmetric by arguing as in [CT16, Proposition 3.1].

Furthermore, the operator $\chi(f)$ is covariant with respect to the action $U$ of the Poincaré group $\mathcal{P}_{+}^{\uparrow}$ on $\mathcal{H}$ that we introduced in Section 3 in the following sense. For a test function $f$ supported in $W_{\mathrm{L}}$ and $(a, \lambda) \in \mathcal{P}_{+}^{\uparrow}$ such that $a \in W_{\mathrm{L}}$, we can show that $\operatorname{Ad} U(a, \lambda)(\chi(f)) \subset$ $\chi\left(f_{(a, \lambda)}\right)$. The key to the proof are the relations (5), see [CT16, Proposition 3.2] for details. 


\section{Weak commutativity}

We introduce the field

$$
\tilde{\phi}(f)=\phi(f)+\chi(f)
$$

and its reflected field $\tilde{\phi}^{\prime}(g)=\phi^{\prime}(g)+\chi^{\prime}(g)=J \tilde{\phi}\left(g_{j}\right) J$ in a similar manner as in [CT16]. For $f$ with support in $W_{\mathrm{L}}$ and such that $f^{*}=f$, the field $\tilde{\phi}(f)$ fulfills the properties listed in [CT16, Proposition 4.1], and a similar result also holds for the reflected field $\tilde{\phi}^{\prime}(g)$. Regarding the domain of $\tilde{\phi}$, we note that, since the domain of $\chi(f)$ contains vectors with finite particle number and with certain analyticity and boundedness properties (see Sec. 4), its domain is included in the domain of $\phi(f)$, and therefore $\operatorname{Dom}(\tilde{\phi}(f))=\operatorname{Dom}(\chi(f))$.

As already mentioned in [CT16], the field $\tilde{\phi}(f)$ has very subtle domain properties. In particular, because of the poles of $S$, after applying this operator to a vector (not the vacuum) in its domain, it generates a vector which is no longer in the domain of $\tilde{\phi}^{\prime}(g)$. For this reason, products of the form $\tilde{\phi}(f) \tilde{\phi}^{\prime}(g)$ and $\tilde{\phi}^{\prime}(g) \tilde{\phi}(f)$ are not well defined, and we need to compute the commutator $\left[\tilde{\phi}(f), \tilde{\phi}^{\prime}(g)\right]$ between arbitrary vectors $\Phi, \Psi$ from a suitable space (see below). Moreover, the commutator is smeared with test functions $f, g$ with only nonzero components corresponding to $b_{1}$.

We start by considering vectors $\Psi_{n}^{\boldsymbol{b}_{\boldsymbol{k}}}$ in the domain discussed in Sec. 4.2. These vectors admit analytic continuation in the first variable, and actually a meromorphic continuation in each variable, to $\pm i \frac{\pi \nu}{2}$. We also note that for certain components $\Psi_{n}^{b_{k_{1}} \cdots b_{k_{n}}}\left(\theta_{1}, \cdots, \theta_{n}\right)$, specifically in the case where two of the indices are equal, $b_{k_{j}}=b_{k_{\ell}}=\alpha$, we can infer the existence of zeros by the following computation:

$$
\begin{aligned}
& \Psi_{n}^{b_{k_{1}} \cdots \alpha \cdots \alpha \cdots b_{k_{n}}}\left(\theta_{1}, \cdots, \theta_{j}, \cdots, \theta_{\ell}, \cdots, \theta_{n}\right) \\
& =\left(\prod_{p=j+1}^{\ell-1} S_{b_{k_{p}} \alpha}\left(\theta_{p}-\theta_{j}\right) S_{\alpha b_{k_{p}}}\left(\theta_{\ell}-\theta_{p}\right)\right) S_{\alpha \alpha}\left(\theta_{\ell}-\theta_{j}\right) \\
& \quad \times \Psi_{n}^{b_{k_{1}} \cdots \alpha \cdots \alpha \cdots b_{k_{n}}}\left(\theta_{1}, \cdots, \theta_{\ell}, \cdots, \theta_{j}, \cdots, \theta_{n}\right) .
\end{aligned}
$$

Hence, by (S7) and (S4), $\Psi_{n}^{b_{k}}$ has a zero at $\theta_{j}-\theta_{\ell}=0$. However, this does not imply existence of zeros for other components. Furthermore, in the proof of Theorem 5.1, we will encounter certain poles of $S$ in the computation. Hence, we consider vectors from the following space:

$\mathcal{D}_{0}:=\left\{\begin{aligned} & \Psi_{n}^{\boldsymbol{b}_{\boldsymbol{k}}} \text { is analytic in } \mathbb{R}^{n}+i\left(-\frac{\pi \nu}{2}, \frac{\pi \nu}{2}\right)^{n}, \\ \Psi \in \mathcal{D}: \quad & \Psi_{n}^{b_{k}}(\boldsymbol{\theta}+i \boldsymbol{\lambda}) \in L^{2}\left(\mathbb{R}^{n}\right) \text { for } \boldsymbol{\lambda} \in\left(-\frac{\pi \nu}{2}, \frac{\pi \nu}{2}\right)^{n}, \text { with a uniform bound and } \\ & \text { has a zero at } \theta_{j}-\theta_{\ell}=0, \pm i \pi(1-\nu), \pm i \pi\left(\frac{3 \nu}{2}-1\right), \pm \frac{i \pi \nu}{2} \text { for all } j, \ell\end{aligned}\right\}$,

where $k_{j}=1,2$. Note that $\mathcal{D}_{0} \subset \operatorname{Dom}(\widetilde{\phi}(f)) \cap \operatorname{Dom}\left(\widetilde{\phi^{\prime}}(g)\right)$.

One can see that $\mathcal{D}_{0}$ is dense as follows: we take

$$
C_{n}(\boldsymbol{\theta}):=\prod_{\lambda \in \Lambda} \prod_{1 \leq j<k \leq n} \frac{\left(\theta_{k}-\theta_{j}-i \lambda\right)\left(\theta_{j}-\theta_{k}-i \lambda\right)}{\left(\theta_{k}-\theta_{j}-2 \pi i\right)\left(\theta_{j}-\theta_{k}-2 \pi i\right)}, \quad \Lambda=\left\{0, \pi(1-\nu), \pi\left(\frac{3 \nu}{2}-1\right), \frac{\pi \nu}{2}\right\},
$$

and consider the set

$$
\left\{M_{C_{n}} P_{n}\left(\xi_{1} \otimes \cdots \otimes \xi_{n}\right), \xi_{j} \in \operatorname{Dom}\left(\chi_{1}(f)\right) \cap \operatorname{Dom}\left(\chi_{1}^{\prime}(g)\right)\right\} .
$$


As $C_{n}$ is symmetric and it has zeros at the poles of $S$, the set above is a subset of $\mathcal{D}_{0}$. Furthermore, as $C_{n}$ is bounded and invertible on $\mathbb{R}^{n}, M_{C_{n}}$ maps a dense set to a dense set. The set $\left\{P_{n}\left(\xi_{1} \otimes \cdots \otimes \xi_{n}\right), \xi_{j} \in \operatorname{Dom}\left(\chi_{1}(f)\right) \cap \operatorname{Dom}\left(\chi_{1}^{\prime}(g)\right)\right\}$ is dense, therefore, so are its image $M_{C_{n}}\left(\operatorname{Dom}(\widetilde{\phi}(f)) \cap \operatorname{Dom}\left(\widetilde{\phi}^{\prime}(g)\right)\right)$ and $\mathcal{D}_{0}$. Thanks to (S8), [Tan16, Proposition E.7] and the properties of $\mathcal{D}_{0}$, we can safely use analytic continuations in the proof of our main theorem.

Theorem 5.1. Let $f$ and $g$ be test functions supported in $W_{\mathrm{L}}$ and $W_{\mathrm{R}}$, respectively, and with the property that $f=f^{*}$ and $g=g^{*}$. Furthermore, assume that $f, g$ have components $f_{b_{k}}=0$ and $g_{b_{k}}=0$ for $k \neq 1$. Then, for each $\Phi, \Psi$ in $\mathcal{D}_{0}$, we have

$$
\left\langle\widetilde{\phi}(f) \Phi, \widetilde{\phi}^{\prime}(g) \Psi\right\rangle=\left\langle\widetilde{\phi}^{\prime}(g) \Phi, \widetilde{\phi}(f) \Psi\right\rangle .
$$

Proof. As in our previous works, we may assume that the vectors $\Phi$ and $\Psi$ are already $S$ symmetric. Furthermore, we recall that the domains of $\widetilde{\phi}(f), \widetilde{\phi}^{\prime}(g)$ coincide with those of $\chi(f), \chi^{\prime}(g)$, respectively, hence we have the following equalities as operators:

$$
\begin{aligned}
& \widetilde{\phi}(f)=\phi(f)+\chi(f)=z^{\dagger}\left(f^{+}\right)+\chi(f)+z\left(J_{1} f^{-}\right), \\
& \widetilde{\phi}^{\prime}(g)=\phi^{\prime}(g)+\chi^{\prime}(g)=z^{\prime \dagger}\left(g^{+}\right)+\chi^{\prime}(g)+z^{\prime}\left(J_{1} g^{-}\right) .
\end{aligned}
$$

Therefore, the (weak) commutator $\left[\widetilde{\phi}(f), \widetilde{\phi^{\prime}}(g)\right]$ expands into several terms that we will compute individually.

The commutator $\left[\phi(f), \phi^{\prime}(g)\right]$

This commutator has been computed in [LS14] and then simplified in the case where $S$ is diagonal in [CT16]. Here, we briefly recall its expression:

$$
\begin{aligned}
& \left(\left[\phi^{\prime}(g), \phi(f)\right] \Psi_{n}\right)^{\boldsymbol{b}_{\boldsymbol{k}}}\left(\theta_{1}, \cdots, \theta_{n}\right) \\
& =\int d \theta^{\prime}\left(g_{b_{1}}^{-}\left(\theta^{\prime}\right)\left(\prod_{p=1}^{n} S_{b_{1} b_{k_{p}}}\left(\theta^{\prime}-\theta_{p}\right)\right) f_{b_{1}}^{+}\left(\theta^{\prime}\right)-g_{b_{1}}^{+}\left(\theta^{\prime}\right)\left(\prod_{p=1}^{n} \overline{S_{b_{1} b_{k_{p}}}\left(\theta^{\prime}-\theta_{p}\right)}\right) f_{b_{1}}^{-}\left(\theta^{\prime}\right)\right) \\
& \quad \times\left(\Psi_{n}\right)^{\boldsymbol{b}_{\boldsymbol{k}}}\left(\theta_{1}, \ldots, \theta_{n}\right) .
\end{aligned}
$$

By (S5) and the analytic properties of $f^{ \pm}, g^{ \pm}$explained in Section 3, the first term in the integrand is equal to the second term up to a shift of $+i \pi$ in $\theta^{\prime}$. Since $S$ has some poles in the physical strip, we obtain residues from this difference.

We are considering test functions $f, g$ whose only non-zero components correspond to $b_{1}$. In this case, the factor $S_{b_{1} b_{k}}$ appearing in the expression of the commutator have exactly two simple poles at $\zeta=i \theta_{b_{1} b_{k}}^{b_{k^{\prime}}}, i \theta_{b_{1} b_{k}}^{b_{k^{\prime}}}$ with $k=1, k^{\prime}=2$ and $k=2, k^{\prime}=1$, as seen in the fusion table in Sec. 2.2.

With the notation $R_{b_{1} b_{k}}^{b_{k^{\prime}}}, R_{b_{1} b_{k}}^{\prime b_{k^{\prime}}}$ which are nonzero only for $k=1, k^{\prime}=2$ and $k=2, k^{\prime}=1$, 
by applying the Cauchy theorem, we get the contributions from the above-mentioned poles:

$$
\begin{aligned}
& \frac{1}{2 \pi i}\left(\left[\phi^{\prime}(g), \phi(f)\right] \Psi_{n}\right)^{b_{k}}\left(\theta_{1}, \ldots, \theta_{n}\right) \\
& =\sum_{k=1,2}\left(\sum_{j=1}^{n} R_{b_{1} b_{j}}^{b_{k}} g_{b_{1}}^{-}\left(\theta_{j}+i \theta_{b_{1} b_{k_{j}}}^{b_{k}}\right) f_{b_{1}}^{+}\left(\theta_{j}+i \theta_{b_{1} b_{k_{j}}}^{b_{k}}\right)\left(\prod_{\substack{p=1 \\
p \neq j}}^{n} S_{b_{1} b_{k_{p}}}\left(\theta_{j}+i \theta_{b_{1} b_{k_{j}}}^{b_{k}}-\theta_{p}\right)\right)\right. \\
& \left.\quad+\sum_{j=1}^{n} R_{b_{1} b_{k_{j}}}^{b_{k}} g_{b_{1}}^{-}\left(\theta_{j}+i \theta_{b_{1} b_{k_{j}}}^{\prime b_{k}}\right) f_{b_{1}}^{+}\left(\theta_{j}+i \theta_{b_{1} b_{k_{j}}}^{\prime b_{k}}\right)\left(\prod_{\substack{p=1 \\
p \neq j}}^{n} S_{b_{1} b_{k_{p}}}\left(\theta_{j}+i \theta_{b_{1} b_{k_{j}}}^{\prime b_{k}}-\theta_{p}\right)\right)\right) \\
& \quad \times\left(\Psi_{n}\right)^{b_{k_{1}} \ldots b_{k_{n}}}\left(\theta_{1}, \ldots, \theta_{n}\right) .
\end{aligned}
$$

More explicitly, the possible terms from the above expression are given by the following.

$$
\begin{aligned}
& \frac{1}{2 \pi i}\left(\left[\phi^{\prime}(g), \phi(f)\right] \Psi_{n}\right)^{b_{k}}\left(\theta_{1}, \ldots, \theta_{n}\right) \\
& =\sum_{j=1}^{n} R_{b_{1} b_{1}}^{b_{2}} g_{b_{1}}^{-}\left(\theta_{j}+i \theta_{b_{1} b_{1}}^{b_{2}}\right) f_{b_{1}}^{+}\left(\theta_{j}+i \theta_{b_{1} b_{1}}^{b_{2}}\right)\left(\prod_{\substack{p=1 \\
p \neq j}}^{n} S_{b_{1} b_{k_{p}}}\left(\theta_{j}+i \theta_{b_{1} b_{1}}^{b_{2}}-\theta_{p}\right)\right) \\
& \quad \times\left(\Psi_{n}\right)^{b_{k_{1}} \ldots b_{1} \ldots b_{k_{n}}}\left(\theta_{1}, \ldots, \theta_{j}, \ldots, \theta_{n}\right) \\
& +\sum_{j=1}^{n} R_{b_{1} b_{2}}^{b_{1}} g_{b_{1}}^{-}\left(\theta_{j}+i \theta_{b_{1} b_{2}}^{\prime b_{1}}\right) f_{b_{1}}^{+}\left(\theta_{j}+i \theta_{b_{1} b_{2}}^{\prime b_{1}}\right)\left(\prod_{\substack{p=1 \\
p \neq j}}^{n} S_{b_{1} b_{k_{p}}}\left(\theta_{j}+i \theta_{b_{1} b_{2}}^{\prime b_{1}}-\theta_{p}\right)\right) \\
& \quad \times\left(\Psi_{n}\right)^{b_{k_{1}} \ldots b_{2} \ldots b_{k_{n}}}\left(\theta_{1}, \ldots, \theta_{j}, \ldots, \theta_{n}\right) \\
& +\sum_{j=1}^{n} R_{b_{1} b_{2}}^{b_{1}} g_{b_{1}}^{-}\left(\theta_{j}+i \theta_{b_{1} b_{2}}^{b_{1}}\right) f_{b_{1}}^{+}\left(\theta_{j}+i \theta_{b_{1} b_{2}}^{b_{1}}\right)\left(\prod_{\substack{p=1 \\
p \neq j}}^{n} S_{b_{1} b_{k_{p}}}\left(\theta_{j}+i \theta_{b_{1} b_{2}}^{b_{1}}-\theta_{p}\right)\right) \\
& \quad \times\left(\Psi_{n}\right)^{b_{k_{1}} \ldots b_{2} \ldots b_{k_{n}}}\left(\theta_{1}, \ldots, \theta_{j}, \ldots, \theta_{n}\right) \\
& +\sum_{j=1}^{n} R_{b_{1} b_{1}}^{b_{2}} g_{b_{1}}^{-}\left(\theta_{j}+i \theta_{b_{1} b_{1}}^{\prime b_{2}}\right) f_{b_{1}}^{+}\left(\theta_{j}+i \theta_{b_{1} b_{1}}^{b_{2}}\right) \\
& \quad \times\left(\prod_{n=1}^{n} S_{b_{1} b_{k_{p}}}\left(\theta_{j}+i \theta_{b_{1} b_{1}}^{\prime b_{2}}-\theta_{p}\right)\right) \\
& \quad \Psi_{p \neq j}^{b_{k_{1}} \ldots b_{1} \ldots b_{k_{n}}}\left(\theta_{1}, \ldots, \theta_{j}, \ldots, \theta_{n}\right) .
\end{aligned}
$$

The commutator $\left[\chi(f), \chi^{\prime}(g)\right]$

We compute this commutator between vectors $\Psi, \Phi$ with only $n$-particle components and with $f, g$ having only non-zero components of type $b_{1}$. Recall the expressions of $\chi(f)$ 
and $\chi^{\prime}(g)$ in Sec. 4, where they are written as the sum of $n$ operators acting on different variables, therefore, there are $n^{2}$ terms in each of the scalar products $\left\langle\chi^{\prime}(g) \Phi, \chi(f) \Psi\right\rangle$ and $\left\langle\chi(f) \Phi, \chi^{\prime}(g) \Psi\right\rangle$. Of these, one can show that the $n(n-1)$ terms in which the above-mentioned operators act on different variables give exactly the same contribution, exactly as in [CT16] (this time the operators $\chi_{1}(f)$ and $\chi_{1}^{\prime}(g)$ are not positive, but $\chi(f) \otimes \mathbb{1} \otimes \cdots \otimes \mathbb{1}$ and $\mathbb{1} \otimes \cdots \otimes \mathbb{1} \otimes \chi_{1}^{\prime}(g)$ are strongly commuting, hence we may consider their polar decomposition), which we denote by $C$, therefore, they cancel in the commutator and hence are irrelevant.

Following [CT16, P. 35], we exhibit the relevant parts $\left(\boldsymbol{k}:=k_{1}, \ldots, k_{n}\right.$ where each $k_{j}$ can take 1,2 .

Furthermore, if $k_{j}=1$, then we put $k_{j}^{\prime}=2$ and if $k_{j}=2$, then $k_{j}^{\prime}=1$ ):

$$
\begin{aligned}
& \left\langle\chi^{\prime}(g) \Phi, \chi(f) \Psi\right\rangle-C \\
& =\sum_{j=1}^{n} \sum_{\substack{k \\
\alpha_{j}, \beta_{j}=1,2}} \int d \theta_{1} \ldots d \theta_{n} \eta_{b_{1} b_{\alpha_{j}}}^{b_{k_{j}}}\left(\prod_{p=1}^{j-1} S_{b_{1} b_{k_{p}}}\left(\theta_{j}-\theta_{p}+i \theta_{\left(b_{1} b_{\alpha_{j}}\right)}^{b_{k_{j}}}\right)\right) f_{b_{1}}^{+}\left(\theta_{j}+i \theta_{\left(b_{1} b_{\alpha_{j}}\right)}^{b_{k_{j}}}\right) \\
& \times\left(\Psi_{n}\right)^{b_{k_{1}} \ldots b_{\alpha_{j}} \ldots b_{k_{n}}}\left(\theta_{1}, \ldots, \theta_{j}-i \theta_{\left(b_{\alpha_{j}} b_{1}\right)}^{b_{k_{j}}}, \ldots, \theta_{n}\right) \eta_{b_{1} b_{\beta_{j}}}^{b_{k_{j}}}\left(\prod_{q=j+1}^{n} S_{b_{1} b_{k_{q}}}\left(\theta_{j}-\theta_{q}+i \theta_{\left(b_{1} b_{\beta_{j}}\right)}^{b_{k_{j}}}\right)\right) \\
& \times g_{b_{1}}^{+}\left(\theta_{j}+i \theta_{\left(b_{1} b_{\beta_{j}}\right)}^{b_{k_{j}}}-i \pi\right) \overline{\left(\Phi_{n}\right)^{b_{k_{1}} \ldots b_{\beta_{j}} \ldots b_{k_{n}}}\left(\theta_{1}, \ldots, \theta_{j}+i \theta_{\left(b_{\beta_{j}} b_{1}\right)}^{b_{k_{j}}}, \ldots, \theta_{n}\right)} \\
& =\sum_{j=1}^{n} \sum_{k} \int d \theta_{1} \ldots d \theta_{n} \eta_{b_{1} b_{k_{j}^{\prime}}}^{b_{k_{j}}}\left(\prod_{p=1}^{j-1} S_{b_{1} b_{k_{p}}}\left(\theta_{j}-\theta_{p}+i \theta_{\left(b_{1} b_{k_{j}^{\prime}}\right)}^{b_{k_{j}}}\right)\right) f_{b_{1}}^{+}\left(\theta_{j}+i \theta_{\left(b_{1} b_{k_{j}^{\prime}}\right.}^{b_{k_{j}}}\right) \\
& \times\left(\Psi_{n}\right)^{b_{k_{1}} \ldots b_{k_{j}^{\prime}} \ldots b_{k_{n}}}\left(\theta_{1}, \ldots, \theta_{j}-i \theta_{\left(b_{k_{j}^{\prime}} b_{1}\right)}^{b_{k_{j}}}, \ldots, \theta_{n}\right) \eta_{b_{1} b_{k_{j}^{\prime}}}^{b_{k_{j}}}\left(\prod_{q=j+1}^{n} S_{b_{1} b_{k_{q}}}\left(\theta_{j}-\theta_{q}+i \theta_{\left(b_{1} b_{k_{j}^{\prime}}\right)}^{b_{k_{j}}}\right)\right) \\
& \times g_{b_{1}}^{+}\left(\theta_{j}+i \theta_{\left(b_{1} b_{k_{j}^{\prime}}\right)}^{b_{k_{j}}}-i \pi\right) \overline{\left(\Phi_{n}\right)^{b_{k_{1}} \ldots b_{k_{j}^{\prime}} \ldots b_{k_{n}}}\left(\theta_{1}, \ldots, \theta_{j}+i \theta_{\left(b_{k_{j}^{\prime}} b_{1}\right)}^{b_{k_{j}}}, \ldots, \theta_{n}\right)} \\
& =\sum_{j=1}^{n} \sum_{k} \int d \theta_{1} \ldots d \theta_{n} \eta_{b_{1} b_{k_{j}^{\prime}}}^{b_{k_{j}}}\left(\prod_{p=1}^{j-1} S_{b_{1} b_{k_{p}}}\left(\theta_{j}-\theta_{p}+i \theta_{b_{1} b_{k_{j}^{\prime}}}^{b_{k_{j}}}\right)\right) f_{b_{1}}^{+}\left(\theta_{j}+i \theta_{b_{1} b_{k_{j}^{\prime}}}^{b_{k_{j}}}\right) \\
& \times\left(\Psi_{n}\right)^{b_{k_{1}} \ldots b_{k_{j}^{\prime}} \ldots b_{k_{n}}}\left(\theta_{1}, \ldots, \theta_{j}, \ldots, \theta_{n}\right) \eta_{b_{1} b_{k_{j}^{\prime}}}^{b_{k_{j}}}\left(\prod_{q=j+1}^{n} S_{b_{1} b_{k_{q}}}\left(\theta_{j}-\theta_{q}+i \theta_{b_{k_{j}^{\prime}}}^{b_{k_{j}}}\right)\right) \\
& \times g_{b_{1}}^{+}\left(\theta_{j}+i \theta_{b_{1} b_{k_{j}^{\prime}}}^{b_{k_{j}}}-i \pi\right) \overline{\left(\Phi_{n}\right)^{b_{k_{1}} \ldots b_{k_{j}^{\prime}} \ldots b_{k_{n}}}\left(\theta_{1}, \ldots, \theta_{j}, \ldots, \theta_{n}\right)},
\end{aligned}
$$

where we used (9) and (10), exploited that $\eta_{b_{1} b_{1}}^{b_{2}}, \eta_{b_{1} b_{2}}^{b_{1}}$ are the only nonzero combinations, then performed the shift $\theta_{j} \rightarrow \theta_{j}+i \theta_{\left(b_{k_{j}^{\prime}} b_{1}\right)}^{b_{k_{j}}}$ in the third equality and used $\theta_{\alpha \beta}^{\gamma}=\theta_{(\alpha \beta)}^{\gamma}+\theta_{(\beta \alpha)}^{\gamma}$. This shift in $\theta_{j}$ is allowed by the analyticity and decay properties of $f^{+}, g^{+}$at infinity in the strip, [CT15, Lemma B.2] and the property of $\Psi, \Phi \in \mathcal{D}_{0}$ explained before Theorem 5.1: more precisely, depending on whether $b_{k_{p}}=b_{1}$ or $b_{2}$ (respectively for $b_{k_{q}}$ ), $S_{b_{1} b_{k_{p}}}(\zeta)$ has a pole at $i \pi \nu$ and $i \pi(1-\nu)$, or at $i \frac{\pi \nu}{2}$ and $i\left(1-\frac{\pi \nu}{2}\right)$, and there are no other poles by the assumptions (S9)(S10). As $\theta_{j} \rightarrow \theta_{j}+i \theta_{\left(b_{k_{j}^{\prime}} b_{1}\right)}^{b_{k_{j}}}=\theta_{j}+i \frac{\pi \nu}{2}$ (this does not depend on $b_{k_{j}}$ : see Table 1 ), the 
integral contour might move across the pole when $\theta_{j} \rightarrow \theta_{j}+i \pi(1-\nu), \theta_{j} \rightarrow \theta_{j}+i \pi\left(\frac{3 \nu}{2}-1\right)$ or $\theta_{j} \rightarrow \theta_{j}+i \frac{\pi \nu}{2}$, depending on the combination of $b_{k_{p}}$ and $b_{k_{j}}$. But these poles are cancelled by the zeros of $\Psi_{n}, \Phi_{n} \in \mathcal{D}_{0}$, hence the shift is legitimate and the result is $L^{1}$ (the integral is the inner product of two $L^{2}$-functions).

Similarly, we can compute the other term $\left\langle\chi(f) \Phi, \chi^{\prime}(g) \Psi\right\rangle$ in the commutator $\left[\chi(f), \chi^{\prime}(g)\right]$ and obtain:

$$
\begin{aligned}
& \left\langle\chi(f) \Phi, \chi^{\prime}(g) \Psi\right\rangle-C \\
& =\sum_{j=1}^{n} \sum_{k} \eta_{b_{1} b_{\alpha_{j}}}^{b_{k_{j}}} \int d \theta_{1} \ldots d \theta_{n} \overline{\left(\prod_{p=1}^{j-1} S_{b_{1} b_{k_{p}}}\left(\theta_{j}-\theta_{p}+i \theta_{\left(b_{1} b_{\alpha_{j}}\right)}^{b_{k_{j}}}\right)\right)} \\
& \alpha_{j}, \beta_{j}=1,2 \\
& \times \overline{f_{b_{1}}^{+}\left(\theta_{j}+i \theta_{\left(b_{1} b_{\alpha_{j}}\right)}^{b_{k_{j}}}\right)\left(\Phi_{n}\right)^{b_{k_{1}} \ldots b_{\alpha_{j}} \ldots b_{k_{n}}}\left(\theta_{1}, \ldots, \theta_{k}-i \theta_{\left(b_{\alpha_{j}} b_{1}\right)}^{b_{k_{j}}}, \ldots, \theta_{n}\right)} \\
& \times \eta_{b_{1} b_{\beta_{j}}}^{b_{k_{j}}}\left(\prod_{q=j+1}^{n} S_{b_{k_{q}} b_{1}}\left(\theta_{q}-\theta_{j}+i \theta_{\left(b_{1} b_{\beta_{j}}\right)}^{b_{k_{j}}}\right)\right) \\
& \times g_{b_{1}}^{+}\left(\theta_{j}-i \theta_{\left(b_{1} b_{\beta_{j}}\right)}^{b_{k_{j}}}\right)\left(\Psi_{n}\right)^{b_{k_{1}} \ldots b_{\beta_{j}} \ldots b_{k_{n}}}\left(\theta_{1}, \ldots, \theta_{j}+i \theta_{\left(b_{\beta_{j}} b_{1}\right)}^{b_{k_{j}}}, \ldots, \theta_{n}\right) \\
& =\sum_{j=1}^{n} \sum_{k} \eta_{b_{1} b_{k_{j}^{\prime}}}^{b_{k_{j}}} \int d \theta_{1} \ldots d \theta_{n}\left(\prod_{p=1}^{j-1} S_{b_{1} b_{k_{p}}}\left(\theta_{j}-\theta_{p}-i \theta_{b_{k_{j}^{\prime}} b_{1}}^{b_{k_{j}}}+i \pi\right)\right) \\
& \times f_{b_{1}}^{+}\left(\theta_{j}-i \theta_{b_{k_{j}^{\prime}} b_{1}}^{b_{k_{j}}}+i \pi\right) \overline{\left(\Phi_{n}\right)^{b_{k_{1}} \ldots b_{k_{j}^{\prime}} \ldots b_{k_{n}}}\left(\theta_{1}, \ldots, \theta_{j}, \ldots, \theta_{n}\right)} \\
& \times \eta_{b_{1} b_{k_{j}^{\prime}}}^{b_{k_{j}}}\left(\prod_{q=j+1}^{n} S_{b_{1} b_{k_{q}}}\left(\theta_{j}-\theta_{q}-i \theta_{b_{1} b_{k_{j}^{\prime}}}^{b_{k_{j}}}+i \pi\right)\right) g_{b_{1}}^{+}\left(\theta_{j}-i \theta_{b_{1} b_{k_{j}^{\prime}}}^{b_{k_{j}}}\right) \\
& \times\left(\Psi_{n}\right)^{b_{k_{1}} \ldots b_{k_{j}^{\prime}} \ldots b_{k_{n}}}\left(\theta_{1}, \ldots, \theta_{j}, \ldots, \theta_{n}\right),
\end{aligned}
$$

where we used (9), (10) and $\theta_{\alpha \beta}^{\gamma}=\theta_{(\alpha \beta)}^{\gamma}+\theta_{(\beta \alpha)}^{\gamma}$, we performed the shift $\theta_{j} \rightarrow \theta_{j}-i \theta_{\left(b_{k_{j}^{\prime}} b_{1}\right)}^{b_{k_{j}}}$ and we used properties (S3)-(S5). As before, we can perform the shift in $\theta_{j}$ using the analyticity and decay properties of $f^{+}, g^{-}$at infinity in the strip, [CT15, Lemma B.2] and the zeros of the vectors $\Psi, \Phi \in \mathcal{D}_{0}$. This also guarantees the fact that the result is still $L^{1}$.

Since there are only two types of fusing processes $\left(b_{1} b_{1}\right) \rightarrow b_{2}$ and $\left(b_{1} b_{2}\right) \rightarrow b_{1}$ in the model, the possible contributions to the expectation values above are

$$
\begin{aligned}
& \left\langle\chi^{\prime}(g) \Phi, \chi(f) \Psi\right\rangle-C \\
& =\sum_{j=1}^{n} \sum_{k} \eta_{b_{1} b_{1}}^{b_{2}} \eta_{b_{1} b_{1}}^{b_{2}} \int d \theta_{1} \ldots d \theta_{n} \prod_{p=1}^{j-1} S_{b_{1} b_{k_{p}}}\left(\theta_{j}-\theta_{p}+i \theta_{b_{1} b_{1}}^{b_{2}}\right) \\
& \times f_{b_{1}}^{+}\left(\theta_{j}+i \theta_{b_{1} b_{1}}^{b_{2}}\right)\left(\Psi_{n}\right)^{b_{k_{1}} \ldots b_{1} \ldots b_{k_{n}}}\left(\theta_{1}, \ldots, \theta_{j}, \ldots, \theta_{n}\right) \prod_{q=j+1}^{n} S_{b_{1} b_{k_{q}}}\left(\theta_{j}-\theta_{q}+i \theta_{b_{1} b_{1}}^{b_{2}}\right) \\
& \times g_{b_{1}}^{+}\left(\theta_{j}+i \theta_{b_{1} b_{1}}^{b_{2}}-i \pi\right) \overline{\left(\Phi_{n}\right)^{b_{k_{1}} \ldots b_{1} \ldots b_{k_{n}}}\left(\theta_{1}, \ldots, \theta_{j}, \ldots, \theta_{n}\right)}
\end{aligned}
$$




$$
\begin{aligned}
& +\sum_{j=1}^{n} \sum_{k} \eta_{b_{1} b_{2}}^{b_{1}} \eta_{b_{1} b_{2}}^{b_{1}} \int d \theta_{1} \ldots d \theta_{n} \prod_{p=1}^{j-1} S_{b_{1} b_{k_{p}}}\left(\theta_{j}-\theta_{p}+i \theta_{b_{1} b_{2}}^{b_{1}}\right) \\
& \times f_{b_{1}}^{+}\left(\theta_{j}+i \theta_{b_{1} b_{2}}^{b_{1}}\right)\left(\Psi_{n}\right)^{b_{k_{1}} \ldots b_{2} \ldots b_{k_{n}}}\left(\theta_{1}, \ldots, \theta_{j}, \ldots, \theta_{n}\right) \prod_{q=j+1}^{n} S_{b_{1} b_{k_{q}}}\left(\theta_{j}-\theta_{q}+i \theta_{b_{2} b_{1}}^{b_{1}}\right) \\
& \times g_{b_{1}}^{+}\left(\theta_{j}+i \theta_{b_{1} b_{2}}^{b_{1}}-i \pi\right) \overline{\left(\Phi_{n}\right)^{b_{k_{1}} \ldots b_{2} \ldots b_{k_{n}}}\left(\theta_{1}, \ldots, \theta_{j}, \ldots, \theta_{n}\right)}
\end{aligned}
$$

and similarly,

$$
\begin{aligned}
& \left\langle\chi(f) \Phi, \chi^{\prime}(g) \Psi\right\rangle-C \\
& =\sum_{j=1}^{n} \sum_{k} \eta_{b_{1} b_{1}}^{b_{2}} \eta_{b_{1} b_{1}}^{b_{2}} \int d \theta_{1} \ldots d \theta_{n} \prod_{p=1}^{j-1} S_{b_{1} b_{k_{p}}}\left(\theta_{j}-\theta_{p}-i \theta_{b_{1} b_{1}}^{b_{2}}+i \pi\right) \\
& \times f_{b_{1}}^{+}\left(\theta_{j}-i \theta_{b_{1} b_{1}}^{b_{2}}+i \pi\right) \overline{\left(\Phi_{n}\right)^{b_{k_{1}} \ldots b_{1} \ldots b_{k_{n}}}\left(\theta_{1}, \ldots, \theta_{j}, \ldots, \theta_{n}\right)} \\
& \times \prod_{q=j+1}^{n} S_{b_{1} b_{k_{q}}}\left(\theta_{j}-\theta_{q}-i \theta_{b_{1} b_{1}}^{b_{2}}+i \pi\right) g_{b_{1}}^{+}\left(\theta_{j}-i \theta_{b_{1} b_{1}}^{b_{2}}\right)\left(\Psi_{n}\right)^{b_{k_{1}} \ldots b_{1} \ldots b_{k_{n}}}\left(\theta_{1}, \ldots, \theta_{j}, \ldots, \theta_{n}\right) \\
& +\sum_{j=1}^{n} \sum_{k} \eta_{b_{1} b_{2}}^{b_{1}} \eta_{b_{1} b_{2}}^{b_{1}} \int d \theta_{1} \ldots d \theta_{n} \prod_{p=1}^{j-1} S_{b_{1} b_{k_{p}}}\left(\theta_{j}-\theta_{p}-i \theta_{b_{2} b_{1}}^{b_{1}}+i \pi\right) \\
& \times f_{b_{1}}^{+}\left(\theta_{j}-i \theta_{b_{2} b_{1}}^{b_{1}}+i \pi\right) \overline{\left(\Phi_{n}\right)^{b_{k_{1}} \ldots b_{2} \ldots b_{k_{n}}}\left(\theta_{1}, \ldots, \theta_{j}, \ldots, \theta_{n}\right)} \\
& \times \prod_{q=j+1}^{n} S_{b_{1} b_{k_{q}}}\left(\theta_{j}-\theta_{q}-i \theta_{b_{1} b_{2}}^{b_{1}}+i \pi\right) g_{b_{1}}^{+}\left(\theta_{j}-i \theta_{b_{1} b_{2}}^{b_{1}}\right)\left(\Psi_{n}\right)^{b_{k_{1}} \ldots b_{2} \ldots b_{k_{n}}}\left(\theta_{1}, \ldots, \theta_{j}, \ldots, \theta_{n}\right) .
\end{aligned}
$$

Now, the commutator $\left[\phi^{\prime}(g), \phi(f)\right]$ cancels the commutator $\left[\chi(f), \chi^{\prime}(g)\right]$ : more precisely, (12a) cancels $(13 \mathrm{a}),(12 \mathrm{~b})$ cancels $(14 \mathrm{~b}),(12 \mathrm{c})$ cancels $(13 \mathrm{~b}),(12 \mathrm{~d})$ cancels $(14 \mathrm{a})$. This uses the following properties:

- The properties of fusion angles and residues, such as $\theta_{b_{1} b_{2}}^{b_{1}}:=\theta_{\left(b_{1} b_{2}\right)}^{b_{1}}+\theta_{\left(b_{2} b_{1}\right)}^{b_{1}}, \theta_{b_{1} b_{1}}^{\prime b_{2}}=$ $\pi-\theta_{b_{1} b_{1}}^{b_{2}}, \theta_{b_{1} b_{2}}^{\prime b_{1}}=\pi-\theta_{b_{2} b_{1}}^{b_{1}}, R_{b_{1} b_{1}}^{\prime b_{2}}=-R_{b_{1} b_{1}}^{b_{2}}$ and $R_{b_{2} b_{1}}^{\prime b_{1}}=-R_{b_{1} b_{2}}^{b_{1}}$.

- Eq. (3) and $R_{b_{1} b_{1}}^{b_{2}}, R_{b_{1} b_{2}}^{b_{1}} \in i \mathbb{R}_{+}$, hence $\left(\eta_{b_{1} b_{1}}^{b_{2}}\right)^{2}=-2 \pi i R_{b_{1} b_{1}}^{b_{2}}$ and $\left(\eta_{b_{1} b_{2}}^{b_{1}}\right)^{2}=-2 \pi i R_{b_{1} b_{2}}^{b_{1}}$.

- $f_{b_{1}}^{+}(\theta+i \pi)=f_{b_{1}}^{-}(\theta), g_{b_{1}}^{+}(\theta-i \pi)=g_{b_{1}}^{-}(\theta)$.

Most of these properties are from Section 2.2.

The commutators $\left[\chi(f), z^{\prime}\left(J_{1} g^{-}\right)\right]$and $\left[z\left(J_{1} f^{-}\right), \chi^{\prime}(g)\right]$

Using the expressions of $\chi(f)$ and $\chi^{\prime}(g)$ in (9) and (10), we can also compute these commutators as in [CT16]. Noting that $\eta_{b_{1} b_{1}}^{b_{2}}, \eta_{b_{1} b_{2}}^{b_{1}}$ are the only possible non-zero combinations, we find

$$
\begin{aligned}
& \left(\left[\chi(f), z^{\prime}\left(J_{1} g^{-}\right)\right] \Psi_{n}\right)^{b_{k_{1}} \ldots b_{k_{n-1}}}\left(\theta_{1}, \cdots, \theta_{n-1}\right) \\
& =\sqrt{n} i \eta_{b_{1} b_{2}}^{b_{1}} \int d \theta^{\prime} g_{b_{1}}^{-}\left(\theta^{\prime}\right) f_{b_{1}}^{+}\left(\theta^{\prime}+i \theta_{\left(b_{1} b_{2}\right)}^{b_{1}}\right)\left(\Psi_{n}\right)^{b_{2} b_{k_{1}} \ldots b_{k_{n-1}}}\left(\theta^{\prime}-i \theta_{\left(b_{2} b_{1}\right)}^{b_{1}}, \theta_{1} \ldots \theta_{n-1}\right)
\end{aligned}
$$




$$
\times\left(\prod_{j=1}^{n-1} S_{b_{1} b_{k_{j}}}\left(\theta^{\prime}-\theta_{j}\right)\right)
$$

which it can be rewritten by shifting $\theta^{\prime} \rightarrow \theta^{\prime}+i \theta_{\left(b_{2} b_{1}\right)}^{b_{1}}$ as follows

$$
\begin{aligned}
& \left(\left[\chi(f), z^{\prime}\left(J_{1} g^{-}\right)\right] \Psi_{n}\right)^{b_{k_{1}} \ldots b_{k_{n-1}}}\left(\theta_{1}, \cdots, \theta_{n-1}\right) \\
& =\sqrt{n} i \eta_{b_{1} b_{2}}^{b_{1}} \int d \theta^{\prime} g_{b_{1}}^{-}\left(\theta^{\prime}+i \theta_{\left(b_{2} b_{1}\right)}^{b_{1}}\right) f_{b_{1}}^{+}\left(\theta^{\prime}+i \theta_{b_{1} b_{2}}^{b_{1}}\right)\left(\Psi_{n}\right)^{b_{2} b_{k_{1}} \ldots b_{k_{n-1}}}\left(\theta^{\prime}, \theta_{1} \ldots \theta_{n-1}\right) \\
& \quad \times\left(\prod_{j=1}^{n-1} S_{b_{1} b_{k_{j}}}\left(\theta^{\prime}+i \theta_{\left(b_{2} b_{1}\right)}^{b_{1}}-\theta_{j}\right)\right) .
\end{aligned}
$$

For the shift in $\theta^{\prime}$, as it is based on an application of the Cauchy Theorem, it uses the analyticity and decay properties of $f^{+}, g^{-}$at infinity in the strip, [CT15, Lemma B.2] and the fact that the poles of the $S$-factors in the product above are cancelled by the zeros of the vector $\Psi_{n} \in \mathcal{D}_{0}$. More precisely, for $b_{k_{j}}=b_{1}, S_{b_{1} b_{1}}(\zeta)$ has a pole at $\zeta=i \pi-i \theta_{b_{1} b_{1}}^{b_{2}}=i \pi(1-\nu)$. Noting that $\pi(1-\nu)<\theta_{\left(b_{2} b_{1}\right)}^{b_{1}}=\frac{\pi \nu}{2}$ for $\frac{2}{3}<\nu<\frac{4}{5}$, the zero of the factor $C_{n}$ at $i \pi\left(1-\frac{3 \nu}{2}\right)$ becomes relevant here (see below (11)), while the pole at $\zeta=i \theta_{b_{1} b_{1}}^{b_{2}}=i \pi \nu$ is not reached by the shift by $\frac{i \pi \nu}{2}$ in $\theta^{\prime}$. The pole of $S_{b_{1} b_{2}}(\zeta)$ at $\zeta=i \frac{\pi \nu}{2}=i \theta_{\left(b_{2} b_{1}\right)}^{b_{1}}$ is cancelled by the zeros of $\Psi_{n}$ arising from $S$-symmetry (see the observations above (11)), as in this case $b_{k_{j}}=b_{2}$, while the pole at $\zeta=i \pi\left(1-\frac{\nu}{2}\right)$ is not reached by the shift by $\frac{i \pi \nu}{2}$ in $\theta^{\prime}$. There are no other poles by the assumptions (S9)(S10).

This also guarantees the fact that the result is still $L^{2}$. Similarly, we have

$$
\begin{aligned}
& \left(\left[z\left(J_{1} f^{-}\right), \chi^{\prime}(g)\right] \Psi_{n}\right)^{b_{k_{1}} \ldots b_{k_{n-1}}}\left(\theta_{1}, \cdots, \theta_{n-1}\right) \\
& =-\sqrt{n} i \eta_{b_{1} b_{2}}^{b_{1}} \int d \theta^{\prime} f_{b_{1}}^{-}\left(\theta^{\prime}\right) g_{b_{1}}^{+}\left(\theta^{\prime}-i \theta_{\left(b_{1} b_{2}\right)}^{b_{1}}\right)\left(\Psi_{n}\right)^{b_{2} b_{k_{1}} \ldots b_{k_{n-1}}}\left(\theta^{\prime}+i \theta_{\left(b_{2} b_{1}\right)}^{b_{1}}, \theta_{1} \ldots \theta_{n-1}\right) \\
& \quad \times\left(\prod_{j=1}^{n-1} S_{b_{k_{j}} b_{1}}\left(\theta_{j}-\theta^{\prime}+i \theta_{\left(b_{1} b_{2}\right)}^{b_{1}}\right)\right),
\end{aligned}
$$

and by shifting $\theta^{\prime} \rightarrow \theta^{\prime}-i \theta_{\left(b_{2} b_{1}\right)}^{b_{1}}$ we can rewrite this expression as

$$
\begin{aligned}
& \left(\left[z\left(J_{1} f^{-}\right), \chi^{\prime}(g)\right] \Psi_{n}\right)^{b_{k_{1}} \ldots b_{k_{n-1}}}\left(\theta_{1}, \cdots, \theta_{n-1}\right) \\
& =-\sqrt{n} i \eta_{b_{1} b_{2}}^{b_{1}} \int d \theta^{\prime} f_{b_{1}}^{-}\left(\theta^{\prime}-i \theta_{\left(b_{2} b_{1}\right)}^{b_{1}}\right) g_{b_{1}}^{+}\left(\theta^{\prime}-i \theta_{b_{1} b_{2}}^{b_{1}}\right)\left(\Psi_{n}\right)^{b_{2} b_{k_{1}} \ldots b_{k_{n-1}}}\left(\theta^{\prime}, \theta_{1} \ldots \theta_{n-1}\right) \\
& \quad \times\left(\prod_{j=1}^{n-1} S_{b_{k_{j}} b_{1}}\left(\theta_{j}-\theta^{\prime}+i \theta_{b_{1} b_{2}}^{b_{1}}\right)\right) \\
& =-\sqrt{n} i \eta_{b_{2} b_{1}}^{b_{1}} \int d \theta^{\prime} f_{b_{1}}^{+}\left(\theta^{\prime}+i \pi-i \theta_{\left(b_{2} b_{1}\right)}^{b_{1}}\right) g_{b_{1}}^{-}\left(\theta^{\prime}+i \pi-i \theta_{b_{1} b_{2}}^{b_{1}}\right)\left(\Psi_{n}\right)^{b_{2} b_{k_{1}} \ldots b_{k_{n-1}}}\left(\theta^{\prime}, \theta_{1} \ldots \theta_{n-1}\right) \\
& \quad \times\left(\prod_{j=1}^{n-1} S_{b_{k_{j}} b_{1}}\left(\theta^{\prime}-\theta_{j}+i \pi-i \theta_{b_{1} b_{2}}^{b_{1}}\right)\right)
\end{aligned}
$$


where we used the property of $f^{-}, g^{+}$under $\pi$-translation and (S5). As before, the shift in $\theta^{\prime}$ is allowed as the poles of the $S$-factors in the product above are cancelled by the zeros of $\Psi_{n} \in \mathcal{D}_{0}$. More precisely, $S_{b_{2} b_{1}}(\zeta)$ has a pole at $i \frac{\pi \nu}{2}$ and this is crossed as $\theta^{\prime}$ is shifted by $i \pi\left(1-\frac{\nu}{2}\right)$, hence the zero of the factor $C_{n}$ at $i \pi(1-\nu)$ becomes relevant, while the pole at $\zeta=i \pi\left(1-\frac{\nu}{2}\right)$ is cancelled by the zeros of $\Psi_{n}$ arising from $S$-symmetry. The pole of $S_{b_{1} b_{1}}(\zeta)$ at $\zeta=i \pi(1-\nu)$ is crossed when $\theta^{\prime}$ is shifted by $i \pi\left(1-\frac{\nu}{2}\right)$, hence we need the zero of the factor $C_{n}$ at $i \frac{\pi \nu}{2}$ to compensate it, while the pole at $\zeta=i \pi \nu$ is not reached by the shift.

The commutators (15) and (16) cancel each other due to the property $\pi-\theta_{b_{1} b_{2}}^{b_{1}}=\theta_{\left(b_{2} b_{1}\right)}^{b_{1}}$ (see Eq. (4)).

The commutators $\left[z^{\dagger}\left(f^{+}\right), \chi^{\prime}(g)\right]$ and $\left[\chi(f), z^{\prime \dagger}\left(g^{+}\right)\right]$

These commutators are the adjoints of the previous ones, therefore, they cancel weakly by the above computations.

This shows the weak-commutativity property of the fields $\tilde{\phi}(f)$ and $\tilde{\phi}^{\prime}(g)$. While being already a major step towards the construction of the model in the algebraic setting, it would be important to obtain a proof of strong commutativity of these fields in order to construct the corresponding wedge-algebras and to prove the existence of strictly local observables through intersection of a shifted right and left wedge. The proof of strong commutativity is however a hard task because of the subtle domain properties of $\tilde{\phi}(f)$ as mentioned at the beginning of Sec. 5. We are in fact able to show that $\tilde{\phi}(f)$ is a symmetric quadratic form on a suitable domain of vectors, but it is not self-adjoint. Therefore, for the proof of strong commutativity, we would need not only to prove existence of self-adjoint extensions of the two fields, but also to select the ones that strongly commute. Some results in this direction are recently available in [Tan15, Tan16] in the case of scalar S-matrices with bound states (e.g. the Bullough-Dodd model), but these techniques are hard to extend to more general S-matrices.

Remark 5.2. Our proof depends only on the properties summarized in Section 2.2 and not on the specific expressions of the S-matrix. This implies that our construction and the proof of weak commutativity work as well if one considers S-matrix such as

$$
S_{b_{k} b_{\ell}}(\zeta)=S_{b_{k} b_{\ell}}^{\mathrm{SG}}(\zeta) \prod_{j=1}^{N} S_{b_{k} b_{\ell}}^{j, \mathrm{CDD}}(\zeta),
$$

where $S_{b_{k} b_{\ell}}^{j, \mathrm{CDD}}(\zeta)$ is a factor as in (1) with (possibly different) parameters $\nu_{j, \pm}$, and $N$ is an odd number (this is necessary to maintain (S11)). Therefore, we have abundant candidates for integrable QFT with the fusion structure considered in this paper.

\section{Concluding remarks}

We have investigated the construction of integrable models with bound states in a series of two papers [CT15, CT16]. In the second paper the construction methods introduced 
in [CT15] are extended to a class of models with several particle species and "diagonal" Smatrices with poles in the physical strip, which includes the $Z(N)$-Ising model and the affineToda field theories as examples. This construction is based on finding observables localized in unbounded wedge-shaped regions to avoid infinite series that characterize strictly local operators. These strictly local observables, with some regularity condition on $S$, should be recovered by taking intersection of the algebras generated by observables in right and left wedges (c.f. [Lec08, AL16]).

Here we considered a model which arises as a deformation of the sine-Gordon model with a parameter $\nu$ which corresponds to a certain range of the coupling constant, $\frac{2}{3}<\nu<\frac{4}{5}$, with an additional CDD factor. As for the proof of weak wedge-locality, we need only some properties of the S-matrix components, and there are abundant examples, as we pointed out in Remark 5.2. As far as we know, that QFTs with such S-matrices have never appeared in the literature. It is an interesting problem to find (or exclude) a Lagrangian description of them (note that the CDD factors appearing here are necessary and our S-matrix cannot be considered as a perturbation of the sine-Gordon model in the sense of, e.g., [SZ16]). In this respect, let us observe that we could find the sign-adjusting CDD factor only for the interval $\frac{2}{3}<\nu<\frac{4}{5}$, while $\nu=1$ corresponds to the (doubled) Ising model. As there is a gap $\frac{4}{5} \leq \nu<1$, this casts doubt that a naive perturbation argument should work.

The resulting theory describes two breathers $b_{1}, b_{2}$ subject to elastic scattering and with the property that they can also fuse to form a bound state (the fusing processes are $\left(b_{1} b_{1}\right) \rightarrow$ $b_{2},\left(b_{1} b_{2}\right) \rightarrow b_{1}$ and $\left.\left(b_{2} b_{1}\right) \rightarrow b_{1}\right)$. This model falls again into the class of "diagonal" Smatrices, and in this sense, it can be regarded as an extension of the previous techniques investigated in [CT16]. This fusion table is the same as the restriction of the table of the Thirring model [Smi92, BFKZ99] to the breather-breather sector (note that it is called "the sine-Gordon model" in the literature in the form factor programme, e.g. [BFKZ99], assuming the equivalence between them). Yet, the original breather-breather S-matrix of the Thirring model does not satisfy the positivity of residues (see Section 2.2), hence cannot be considered as a separate model. In this sense, the present paper highlights the really necessary properties of the S-matrix for wedge-locality and contains a new hint in the construction of interacting quantum field theories in the algebraic framework.

An interesting problem would be an extension of such a construction to integrable models with "non-diagonal" S-matrices, e.g. the Thirring model [BFKZ99] or SU( $N)$-invariant Smatrices [BFK08]. It would be interesting to show that weak wedge-commutativity holds at least for some of these models. They are currently under investigation. It should be noted that commutation relations of pointlike fields have not been proved for these models ${ }^{8}$. Our methods represent a complementary way of proving existence of local observables, which may work if the S-matrix components concerning elementary particles (solitons in the case of the Thirring model) have only simple poles, yet here several analytic questions (such as the domains of unbounded operators and modular nuclearity) must be addressed.

On the other hand, there are some factorizing S-matrices which do not satisfy the assumption (S10) or an analogous assumption [BCDS90, Section 4.2.4]. Constructing observables for such models, or in more generally, observables generating particles with higher-poles in the S-matrix components is an interesting open problem.

\footnotetext{
${ }^{8}$ Michael Karowski, private communication.
} 


\begin{tabular}{|c|c|l|}
\hline Range of $\nu$ & The residue of pole of $S_{11}^{11}$ & Comment \\
\hline $4 / 5<\nu<1$ & $-i \mathbb{R}_{+}$ & No adjusting CDD factor found \\
\hline $2 / 3<\nu<4 / 5$ & $-i \mathbb{R}_{+}$ & Adjusting CDD factors found \\
\hline $1 / 2<\nu<2 / 3$ & $-i \mathbb{R}_{+}$ & $\begin{array}{l}\text { There are three breathers if one requires the } \\
\text { maximal analyticity within breathers. } \\
\text { No adjusting CDD factor found }\end{array}$ \\
\hline $0<\nu<1 / 2$ & $i \mathbb{R}_{+}$ & $\begin{array}{l}\text { There is a breather } b_{K} \text { for which } \\
\operatorname{Res}_{\zeta=i b_{b_{1} b_{k}}} S_{b_{1} b_{K}}(\zeta) \in-i \mathbb{R}_{+}\end{array}$ \\
\hline
\end{tabular}

Table 2: Ranges of the coupling constant $\nu$ in the sine-Gordon model

As we mentioned in Section 2.2, the S-matrix studied in the present paper is a deformation of the S-matrix of the sine-Gordon model in the range of the coupling constant $\frac{2}{3}<\nu<\frac{4}{5}$ by a CDD factor. The reason for the CDD factor is the following: while the fusion table of the breather-breather S-matrix is closed under fusions, these S-matrix components cannot be considered as a separate model because the residues of some poles in the physical strip are on $-i \mathbb{R}_{+}$(see comment before Eq. (3)), which is not compatible with our proof. We note that also in the proof of local commutativity theorem in the form factor programme [Que99] this property is used, therefore, it must be adjusted in some way. Varying the range of the coupling constant $\nu$, the situation is as pictured in Table 2. In particular, as explained in Sec. 2.2, for $\frac{4}{5}<\nu<1$ there are no values of $\nu_{-}$and $\nu_{+}$which fulfill the required conditions after Eq. (2), and our simplest form for a CDD factor does not work. For $\frac{1}{2}<\nu<\frac{2}{3}$ there are three breathers in the model (if we take the maximal analyticity literally), and both $S^{\mathrm{SG}}{ }_{11}$ and $S^{\mathrm{SG}}{ }_{12}$ have $s$-channel poles with residues in $-i \mathbb{R}_{+}$. We could not find a suitable CDD factor adjusting all the residues. Finally, in the range $0<\nu<\frac{1}{2}$ there is an increasing number of breathers by maximal analyticity, and while Res ${ }_{\zeta=i \theta_{b_{1} b_{1}}^{b_{2}}} S^{\mathrm{SG}_{11}^{2}}(\zeta) \in i \mathbb{R}_{+}$, there are other S-matrix components whose residues are in $-i \mathbb{R}_{+}$. We could not find a suitable CDD factor for this range as well.

Finally, the domain of the operator $\chi(f)$ is considerably small. Indeed, one can show that even the one-particle components $\chi_{1}(f)$ is not self-adjoint, see [Tan15]; moreover, the domains of $\chi_{n}(f)$ must be somehow enlarged compensating the factor $C_{n}$. We believe that these domain issues are fundamentally related with the complicated fusing processes of the models, hence deserve a separate study.

\section{Acknowledgements}

We thank Michael Karowski for informing us of the current status of the form factor programme. Y.T. thanks Sabina Alazzawi and Wojciech Dybalski for the discussion on the relations between Thirring and sine-Gordon models.

Y.T. is supported by the JSPS overseas fellowship. 


\section{References}

[Ala14] Sabina Alazzawi. Deformations of quantum field theories and the construction of interacting models. 2014. Ph.D. thesis, Universität Wien, http://arxiv.org/abs/1503. 00897.

[AL16] Sabina Alazzawi and Gandalf Lechner. Inverse scattering and locality in integrable quantum field theories. 2016. https://arxiv.org/abs/1608.02359.

[BFKZ99] H. Babujian, A. Fring, M. Karowski, and A. Zapletal. Exact form factors in integrable quantum field theories: the sine-Gordon model. Nuclear Phys. B, 538(3):535-586, 1999. http://arxiv.org/abs/hep-th/9805185.

[BK02] H. Babujian and M. Karowski. Sine-Gordon breather form factors and quantum field equations. J. Phys. A, 35(43):9081-9104, 2002.

[BFK08] H. Babujian, A. Foerster, and M. Karowski. The nested SU(N) off-shell Bethe ansatz and exact form factors. J. Phys. A, 41(27):275202, 21, 2008. https://arxiv.org/abs/ hep-th/0611012v1.

[BCDS90] H. W. Braden, E. Corrigan, P. E. Dorey, and R. Sasaki. Affine Toda field theory and exact $S$-matrices. Nuclear Phys. B, 338(3):689-746, 1990. https://doi.org/10.1016/ 0550-3213 (90) 90648-W.

[BR16] Dorothea Bahns and Kasia Rejzner. The quantum sine Gordon model in perturbative AQFT. 2016. https://arxiv.org/abs/1609.08530.

[BFM07] G. Benfatto, P. Falco, and V. Mastropietro. Functional integral construction of the massive thirring model: Verification of axioms and massless limit. Commun. Math. Phys, 273(1):67-118, 2007.

[BFM09] G. Benfatto, P. Falco, and V. Mastropietro. Massless sine-Gordon and massive Thirring models: Proof of the Coleman's equivalence. Commun. Math. Phys., 285: 713-762, 2009.

[BT13] Marcel Bischoff and Yoh Tanimoto. Construction of Wedge-Local Nets of Observables through Longo-Witten Endomorphisms. II. Comm. Math. Phys., 317(3):667-695, 2013. http://arxiv.org/abs/1111.1671.

[BT15] Marcel Bischoff and Yoh Tanimoto. Integrable QFT and Longo-Witten endomorphisms. Ann. Henri Poincaré, 16(2):569-608, 2015. http://arxiv.org/abs/1305.2171.

[CT15] Daniela Cadamuro and Yoh Tanimoto. Wedge-Local Fields in Integrable Models with Bound States. Comm. Math. Phys., 340(2):661-697, 2015. http://arxiv.org/abs/ 1502.01313.

[CT16] Daniela Cadamuro and Yoh Tanimoto. Wedge-local fields in integrable models with bound states II. diagonal S-matrix. 2016. to appear in Ann. Henri Poincaré, http://arxiv.org/abs/1601.07092.

[DT11] Wojciech Dybalski and Yoh Tanimoto. Asymptotic completeness in a class of massless relativistic quantum field theories. Comm. Math. Phys., 305(2):427-440, 2011. http: //arxiv.org/abs/1006.5430. 
[FS76] Jürg Fröhlich and Erhard Seiler. The massive Thirring-Schwinger model $\left(\mathrm{QED}_{2}\right)$ : convergence of perturbation theory and particle structure. Helv. Phys. Acta, 49(6):889-924, 1976.

[Lec03] Gandalf Lechner. Polarization-free quantum fields and interaction. Lett. Math. Phys., 64(2):137-154, 2003. http://arxiv.org/abs/hep-th/0303062.

[Lec08] Gandalf Lechner. Construction of quantum field theories with factorizing $S$-matrices. Comm. Math. Phys., 277(3):821-860, 2008. http://arxiv.org/abs/math-ph/0601022.

[Lec15] Gandalf Lechner. Algebraic constructive quantum field theory: Integrable models and deformation techniques. In Advances in Algebraic Quantum Field Theory, pages 397448. Springer International Publishing, New York-London-Paris, 2015. https://arxiv . org/abs/1503.03822.

[LS14] Gandalf Lechner and Christian Schützenhofer. Towards an operator-algebraic construction of integrable global gauge theories. Ann. Henri Poincaré, 15(4):645-678, 2014. http://arxiv.org/abs/1208.2366.

[LST13] Gandalf Lechner, Jan Schlemmer, and Yoh Tanimoto. On the equivalence of two deformation schemes in quantum field theory. Lett. Math. Phys., 103(4):421-437, 2013. http://arxiv.org/abs/1209.2547.

[Par77] Yong Moon Park. Massless quantum sinegordon equation in two spacetime dimensions: Correlation inequalities and infinite volume limit. Journal of Mathematical Physics, 18(12):2423-2426, 1977.

[Que99] Thomas Quella. Formfactors and locality in integrable models of quantum field theory in 1+1 dimensions (in German). 1999. Diploma thesis, Freie Universität Berlin.

http://www.thp.uni-koeln.de/ tquella/1999QuellaDiploma.pdf.

[Sch97] Bert Schroer. Modular localization and the bootstrap-formfactor program. Nuclear Phys. B, 499(3):547-568, 1997. http://arxiv.org/abs/hep-th/9702145.

[Smi92] F.A. Smirnov. Form factors in completely integrable models of quantum field theory, volume 14 of Advanced Series in Mathematical Physics. World Scientific Publishing Co. Inc., River Edge, NJ, 1992.

[SZ16] F.A. Smirnov and A.B. Zamolodchikov. On space of integrable quantum field theories. 2016. https://arxiv.org/abs/1608.05499.

[Tan12] Yoh Tanimoto. Construction of Wedge-Local Nets of Observables Through Longo-Witten Endomorphisms. Comm. Math. Phys., 314(2):443-469, 2012. http://arxiv.org/abs/ 1107.2629.

[Tan14] Yoh Tanimoto. Construction of two-dimensional quantum field models through LongoWitten endomorphisms. Forum Math. Sigma, 2:e7, 31, 2014. http://arxiv.org/abs/ 1301.6090 .

[Tan15] Yoh Tanimoto. Self-adjointness of bound state operators in integrable quantum field theory. 2015. http://arxiv.org/abs/1508.06402. 
[Tan16] Yoh Tanimoto. Bound state operators and wedge-locality in integrable quantum field theories. SIGMA Symmetry Integrability Geom. Methods Appl., 12:100, 39 pages, 2016. https://arxiv.org/abs/1602.04696.

[ZZ79] Alexander B. Zamolodchikov and Alexey B. Zamolodchikov. Factorized S-matrices in two dimensions as the exact solutions of certain relativistic quantum field theory models. Ann. Physics, 120(2):253-291, 1979. 